Check for updates

Cite this: Phys. Chem. Chem. Phys. 2021, 23, 7586

Received 23rd July 2020,

Accepted 19th October 2020

DOI: $10.1039 / d 0 c p 03929 b$

rsc.li/pccp

\section{Time-dependent quantum mechanical wave packet dynamics + t}

\author{
Narayanasami Sathyamurthy (iD *a and Susanta Mahapatra (iD b
}

Starting from a model study of the collinear $\left(\mathrm{H}_{1} \mathrm{H}_{2}\right)$ exchange reaction in 1959, the time-dependent quantum mechanical wave packet (TDQMWP) method has come a long way in dealing with systems as large as $\mathrm{Cl}+\mathrm{CH}_{4}$. The fast Fourier transform method for evaluating the second order spatial derivative of the wave function and split-operator method or Chebyshev polynomial expansion for determining the time evolution of the wave function for the system have made the approach highly accurate from a practical point of view. The TDQMWP methodology has been able to predict state-to-state differential and integral reaction cross sections accurately, in agreement with available experimental results for three dimensional $\left(H_{1} H_{2}\right)$ collisions, and identify reactive scattering resonances too. It has become a practical computational tool in predicting the observables for many $\mathrm{A}+\mathrm{BC}$ exchange reactions in three dimensions and a number of larger systems. It is equally amenable to determining the bound and quasi-bound states for a variety of molecular systems. Just as it is able to deal with dissociative processes (without involving basis set expansion), it is able to deal with multi-mode nonadiabatic dynamics in multiple electronic states with equal ease. We present an overview of the method and its strength and limitations, citing examples largely from our own research groups.

\footnotetext{
${ }^{a}$ Indian Institute of Science Education and Research Mohali, SAS Nagar, 140306, India. E-mail: nsathyamurthy@gmail.com

${ }^{b}$ School of Chemistry, University of Hyderabad, Hyderabad, 500 046, India. E-mail: susanta.mahapatra@uohyd.ac.in

$\dagger$ Dedicated to J. P. Toennies on his 90th birthday.

¥ Electronic supplementary information (ESI) available: A graphic illustration of the time evolution of a wave packet in scaled and skewed coordinates for the collinear $\left(\mathrm{He}, \mathrm{H}_{2}{ }^{+}\right)$exchange reaction and that in Jacobi coordinates for $3 \mathrm{D}(\mathrm{He}$, $\mathrm{H}_{2}^{+}$) collisions. See DOI: 10.1039/d0cp03929b
}

\section{Introduction}

Some time ago, when a friend of ours was looking at our results obtained from an investigation of time-dependent quantum mechanical wave packet (TDQMWP) dynamics by solving the time-dependent Schrödinger equation (TDSE) on a three dimensional (3D) grid, he questioned the wisdom of adding another dimension (time, $t$ ) to the difficult problem when one

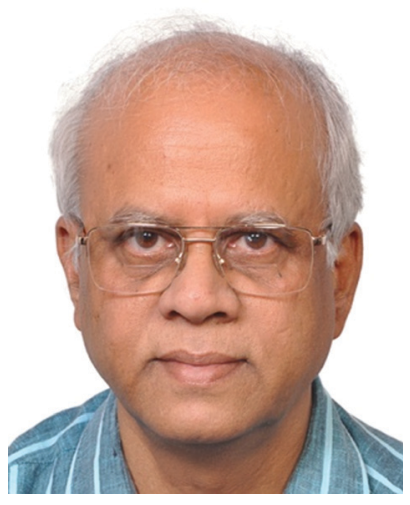

Narayanasami Sathyamurthy
Dr N. Sathyamurthy, Honorary Professor at the Indian Institute of Science Education and Research (IISER) Mohali, is a Fellow of the Indian Academy of Sciences, Bengaluru, Indian National Science Academy, New Delhi, and The World Academy of Sciences, Trieste, Italy. A recipient of several awards and honors in India, he has authored/ coauthored more than 200 research publications in the area of molecular reaction dynamics and atomic and molecular clusters. He is currently the Chief Editor, Resonance - Journal of Science Education, published by the Indian Academy of Sciences, Bengaluru, and an Editor or a member of the Editorial Board of a few other journals.

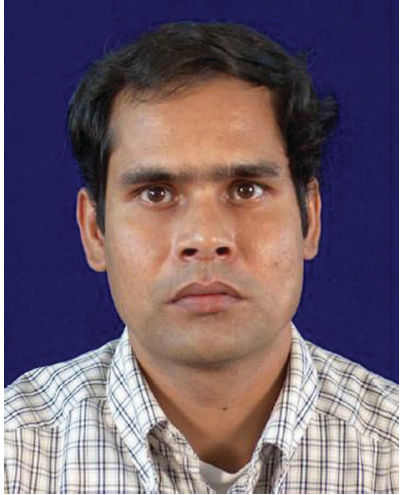

Susanta Mahapatra
Dr S. Mahapatra, Senior Professor at the School of Chemistry, University of Hyderabad, Hyderabad, India, is a Fellow of the Indian Academy of Sciences, Bengaluru, National Academy of Sciences, India, and The Andhra Pradesh-Telangana State Academy of Sciences, India. A recipient of several awards and honors in India and abroad, he has authored/ coauthored more than 130 research publications in the area of nonadiabatic chemical dynamics. He is an Associate Editor of Resonance - Journal of Science Education, published by the Indian Academy of Sciences, Bengaluru. 
could get all the information (experimental observables) by solving the time-independent Schrödinger equation (TISE). What he did not say, but we guess he implied, was that Schrödinger himself solved the stationary state problems of the hydrogen atom, ${ }^{1}$ the harmonic oscillator, the rigid rotor and diatomic molecules ${ }^{2}$ before looking at scattering processes and the like in the 4 th paper in the series. ${ }^{3}$ In some sense, the question of our friend was like asking what the need was to watch a football game when all one needed to know was the final score!

Schrödinger seemed to have been interested in addressing only the conceptual difficulties in his papers as he believed that the mathematical aspects were easily solvable. Dirac, ${ }^{4}$ however, was more pragmatic. He declared, "The fundamental laws necessary for the mathematical treatment of a large part of physics and the whole of chemistry are thus completely known and the difficulty lies only in the fact that application of these laws leads to equations that are too complex to be solved." Numerical solutions were far away in the future as computers were yet to be invented.

From a formal point of view, the TISE is an eigenvalue problem, while the TDSE is an initial value problem. The latter has an advantage in that it can include continuum states with equal ease and basis set convergence would not be an issue, which means that collision induced dissociation, photodissociation and similar processes on solid/liquid surfaces can be treated with ease using the time-dependent quantum mechanical (TDQM) approach. It took nearly three decades after the publication of Schrödinger's papers for the first numerical solutions to be obtained using computers Illiac and IBM-704 by Mazur and Rubin. ${ }^{5}$ The work was path breaking in that it computed the rate constant for a collinear $\left(\mathrm{H}, \mathrm{H}_{2}\right)$ exchange reaction using a model potential energy surface (PES) by solving the TDSE for the nuclear motion. It also laid the foundation for carrying out wave packet (WP) dynamics on one dimensional (1D) and two dimensional (2D) grids.

McCullough and Wyatt ${ }^{6}$ used the finite difference method for evaluating the second derivative of the wave function with respect to the spatial coordinates and finite difference and explicit methods for evaluating the first derivative of the wave function with respect to time for the collinear $\left(\mathrm{H}, \mathrm{H}_{2}\right)$ exchange reaction on a realistic PES. They were perhaps the best possible numerical approaches at that time, with the then available computational resources.

Glen E. Kellerhals, ${ }^{7}$ a senior $\mathrm{PhD}$ scholar in the research group of Professor Lionel M. Raff at Oklahoma State University, had estimated that he had spent US \$40000 worth of computer time on an IBM system 360/65 machine in solving model collinear $(\mathrm{A}+\mathrm{BC})$ exchange reactions for completing his $\mathrm{PhD}$ thesis work in 1974. In contrast, Aditya Narayan Panda, ${ }^{8}$ a PhD student at the Indian Institute of Technology, Kanpur, in 2004 could compute the reaction probability values for the $\left(\mathrm{He}, \mathrm{H}_{2}{ }^{+}\right)$ exchange reaction in three dimensions (for total angular momentum $J=0$ ) using the TDQMWP methodology on a Pentium IV machine with $256 \mathrm{MB}$ RAM in about $4.5 \mathrm{~h}$. In 2012, Sujitha Kolakkondy, ${ }^{9}$ another PhD student in the same lab, was able to carry out TDQMWP calculations for $\left(\mathrm{He}, \mathrm{H}_{2}{ }^{+}\right)$ reactive scattering in three dimensions for a number of $J(=0-70)$ $(K=0-10)$ states and compute reaction cross section values over a range of translational energy $\left(E_{\text {trans }}=0-4.7 \mathrm{eV}\right)$ by using PCs and workstations in the lab. Such a development has been possible largely due to larger computer memory and increased computing speed becoming readily available in recent decades. Levitt has pointed out elsewhere ${ }^{10}$ how the price of computers and their physical size have come down (from a main frame computer occupying a large air conditioned hall to a laptop) by four orders of magnitude while the available computer memory and the CPU (central processing unit) speed have increased by four orders of magnitude, during the same period 1967-2013.

As decades went by and the time resolution of the experimental investigation of elementary chemical processes improved from milliseconds to micro-, nano-, pico- and femtoseconds, it made more sense to look at the time evolution of chemical dynamical systems. ${ }^{11,12}$ Although our thinking is "classical", the dynamical processes at the atomic/molecular level are quantum mechanical in nature. ${ }^{13}$

Interestingly, quasi-classical trajectory (QCT) calculations were also initiated ${ }^{14}$ at about the same time as TDQM calculations and the former made much more rapid progress in modelling many elementary chemical reactions in the following years. ${ }^{15}$ Molecular dynamics (MD) simulations have become common and have been extended to large biomolecular systems in recent years. ${ }^{10}$

While QCT calculations accounted for much of the averaged observables in state-selected and state-to-state chemistry for a number of systems, quantal effects like resonances could only be accounted for through detailed time-independent quantum mechanical (TIQM) and TDQM calculations. ${ }^{16,17}$

Thanks to the sustained efforts of chemists in probing the transition state, "capturing" the transition state ${ }^{18,19}$ and identifying reactive scattering resonances experimentally ${ }^{20,21}$ became a reality. As more efforts went into "controlling" chemical reactions dynamically (rather than passively through initial state selection) in real time using laser pulses, ${ }^{22-25}$ it made more sense to follow a TDQM approach to investigate elementary chemical reactions.

Photo-excitation/dissociation processes also lent themselves to a dynamical description in real time. ${ }^{26}$ Increased experimentation in femtochemistry meant increased use of the timedependent approach. These developments also warranted the inclusion of more than one electronic state and nonadiabatic coupling in dynamical investigations. Recent developments in the use of attosecond pulses in probing atomic and molecular processes $^{27}$ require dealing with multi-electronic state dynamical processes in real time. Our earlier write $\mathrm{ups}^{28-32}$ and those of others ${ }^{33-37}$ on the TDQMWP approach outlined the motive behind the method and the developments in the methodology till then.

We present an overview of subsequent developments in the field and indicate the likely future directions for the area of research. Since this is not a review article, we do not review all the research papers published in the field. We restrict ourselves largely to what is called the grid method in this perspective, 
although we do point out the developments in combining it with other methods.

\section{Theoretical background}

Any problem in non-relativistic physics, chemistry and biology can, in principle, be solved by solving the TDSE

$$
\hat{H} \Psi=i \hbar \frac{\partial \Psi}{\partial t}
$$

where $\hat{H}$ is the Hamiltonian of the system under consideration and $\Psi$ is the wave function. The basic postulate of quantum mechanics states that the wave function contains all the information about the system. It is up to us to extract it.

The TDQMWP approach is conceptually simple to adopt and straightforward to implement. It is easy to interpret the dynamical outcomes and to compare the results with (experimental) observables. It consists of (i) defining the initial state of the system in terms of the wave function, $\Psi(0)$, for example, on a grid in suitable coordinates, (ii) evolving the wave function, $\Psi(t)$, with time, given the Hamiltonian of the system and (iii) analysing the wave function at different time intervals to obtain insight into the dynamics and computing the observables for comparing with experimental results at the "end" of the time evolution. The size of the grid in the TDQMWP method depends on the choice of problem under investigation. For vibrational spectra of a diatomic molecule in its low vibrational states, for example, it is enough to concentrate on a set of grid points along the internuclear distance $(r)$ in the vicinity of the potential energy minimum. For investigating associative/ dissociative processes, one needs to consider grid points over an extended range of $r$. While the magnitude of $\mathrm{d} V / \mathrm{d} r$ (force) varies gradually with a variation in $r$ for large $r$, it varies dramatically for small $r$. Therefore, in principle, one can choose a coarse mesh for large $r$ and a fine mesh for small $r$. In practice, this is not easily implementable. Some authors ${ }^{38}$ have used a floating grid. But this is not simple. Unless one is careful, the results may not be time reversible. Choi and Vaníček ${ }^{39}$ have come up with a strategy to include the spatial grid in the time evolution, to make sure that the evolution is time reversible.

While one can use a large number (64/128/256/512) of grid points in one dimension, it becomes difficult to keep the number of grid points large in higher dimensions. While an $\mathrm{A}+\mathrm{BC}$ exchange reaction in three dimensions calls for the use of three variables, larger polyatomic systems require TDQM investigations in larger dimensions. That explains why it took time for the TDQMWP method to become a practical tool in the study of chemical reactions and why it was restricted initially to systems involving very few atoms. Before getting into the details of the above mentioned three steps in the grid method, it is important to discuss the difficulties involved in formulating the potential energy surface (PES) for the system under consideration and the choice of coordinates. For a diatomic molecule (or ionic species) in its ground electronic state, there is only one coordinate that the potential energy depends upon, that is, $r$. For polyatomic molecules, there are $3 n-5(6)$ vibrational degrees of freedom, depending upon whether the molecule is linear or nonlinear. For small amplitude motions, normal coordinates $^{40}$ might suffice. But for large amplitude motions and for events involving bond breaking and bond forming, some other coordinates have to be used.

For a reactive $\mathrm{A}+\mathrm{BC}$ (triatomic) system in collinear geometry, scaled and skewed coordinates $(x, y)$ were developed long ago ${ }^{41}$ to describe the motion equivalently to that of a single particle of mass $\mu=\frac{m_{\mathrm{A}}\left(m_{\mathrm{B}}+m_{\mathrm{C}}\right)}{\left(m_{\mathrm{A}}+m_{\mathrm{B}}+m_{\mathrm{C}}\right)}$ in $(x, y)$ space, where

$$
\begin{gathered}
x=r_{\mathrm{AB}}+\beta r_{\mathrm{BC}} \sin \theta \\
y=\beta r_{2} \cos \theta,
\end{gathered}
$$

with

$$
\beta=\left[\frac{m_{\mathrm{C}}\left(m_{\mathrm{A}}+m_{\mathrm{B}}\right)}{m_{\mathrm{A}}\left(m_{\mathrm{B}}+m_{\mathrm{C}}\right)}\right]^{\frac{1}{2}}
$$

and

$$
\theta=\sin ^{-1}\left[\frac{m_{\mathrm{A}} m_{\mathrm{C}}}{\left(m_{\mathrm{A}}+m_{\mathrm{B}}\right)\left(m_{\mathrm{B}}+m_{\mathrm{C}}\right)}\right]^{\frac{1}{2}},
$$

where $m_{\mathrm{A}}, m_{\mathrm{B}}$ and $m_{\mathrm{C}}$ represent the masses of atoms $\mathrm{A}, \mathrm{B}$, and $\mathrm{C}$, respectively.

For noncollinear geometries, one could use the mass-scaled Jacobi coordinates $(R, r, \gamma)$ illustrated in Fig. 1 . It is evident that there is more than one possible set of Jacobi coordinates, depending upon the arrangement channel. The difficulty in choosing the right set of Jacobi coordinates at different stages of a collision event can be circumvented by working in Delves coordinates ${ }^{42}$ in two dimensions and in hyperspherical coordinates $(\rho, \theta, \phi)$ in three dimensions. The latter coordinates are

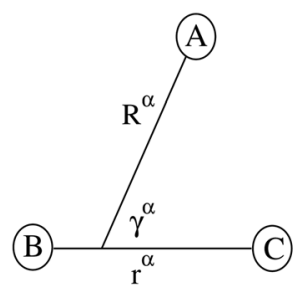

(a)

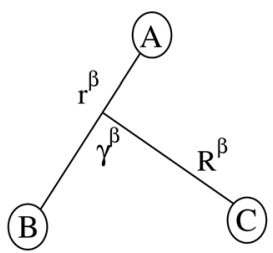

(b)

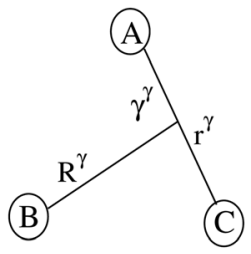

(c)

Fig. 1 The Jacobi coordinates for the $\alpha, \beta$ and $\gamma$ arrangement channels of the $\mathrm{A}+\mathrm{BC}$ collisional system. 
defined in many different ways in the literature. ${ }^{43-47}$ However, the most often used ones are due to Morse and Feshbach, ${ }^{43}$ who defined them in terms of the Jacobi vectors $\vec{R}$ and $\vec{r}$ as

$$
\begin{gathered}
\rho=\left(\vec{r}^{2}+\vec{R}^{2}\right)^{1 / 2} \\
\theta=\tan ^{-1}\left(\frac{r}{R}\right)
\end{gathered}
$$

and

$$
\phi=\cos ^{-1}\left(\frac{\vec{r} \cdot \vec{R}}{r R}\right) .
$$

While the hyperradius $(\rho)$ is unique in all definitions, the hyperangles $(\theta$ and $\phi)$ are not. For larger systems like A + BCD, $\mathrm{AB}+\mathrm{CD}$, etc., one needs to adopt suitable coordinates for investigating the chemical reactions involved.

The Hamiltonian can be written in any of the stated coordinates for a given molecular process. For example, the Hamiltonian of the A + BC collisional system in the reactant channel $(\alpha$-channel in Fig. 1) mass-scaled body-fixed Jacobi coordinates $(R, r, \gamma)$ is given by ${ }^{48-51}$

$$
\begin{aligned}
\hat{H}= & {\left[-\frac{\hbar^{2}}{2 \mu^{\prime}}\left(\frac{\partial^{2}}{\partial R^{2}}+\frac{\partial^{2}}{\partial r^{2}}\right)+\frac{\dot{j}^{2}}{2 I}+\frac{\hbar^{2}}{2 \mu^{\prime} R^{2}}\left[\boldsymbol{J}^{2}-2 K^{2}\right]+V(R, r, \gamma)\right] \delta_{K, K} } \\
& -\frac{\hbar^{2}}{2 \mu^{\prime} R^{2}} \lambda_{J K}^{+}\left(\frac{\partial}{\partial \gamma}-K \cot \gamma\right) \delta_{K, K+1} \\
& -\frac{\hbar^{2}}{2 \mu^{\prime} R^{2}} \lambda_{J K}^{-}\left(-\frac{\partial}{\partial \gamma}-K \cot \gamma\right) \delta_{K, K-1} .
\end{aligned}
$$

In the above equation, $\boldsymbol{j}$ is the rotational angular momentum operator of the BC diatom, $\boldsymbol{J}$ is the total (three-body) angular momentum operator, $V(R, r, \gamma)$ is the interaction potential, $\mu^{\prime}=\sqrt{\frac{m_{\mathrm{A}} m_{\mathrm{B}} m_{\mathrm{C}}}{m_{\mathrm{A}}+m_{\mathrm{B}}+m_{\mathrm{C}}}}$ is the three-body scaled reduced mass and $I=\frac{\mu^{\prime} R^{2} r^{2}}{R^{2}+r^{2}}$ is the three-body moment of inertia. The quantity $K=-J, \ldots, 0, \ldots,+J$ is the projection of $J$ and also $j$ on the body-fixed $Z$-axis and $\lambda_{J K}^{ \pm}=\sqrt{J(J+1)-K(K \pm 1)}$. The last two off-diagonal terms in eqn (9) couple the various $K$ states in the body-fixed frame and are known as Coriolis coupling terms. ${ }^{52}$ When these terms are neglected, one arrives at the well-known coupled-states or centrifugal sudden (CS) approximation. ${ }^{53,54}$ Within this approximation $K$ is a good quantum number and is conserved in the body-fixed Jacobi frame. This approximation simplifies the $J \neq 0$ calculations by reducing the dimensionality of the problem from four to three.

Generating PESs of chemical accuracy $\left( \pm 1 \mathrm{kcal} \mathrm{mol}^{-1}\right)$ or of spectroscopic accuracy $\left( \pm 1 \mathrm{~cm}^{-1}\right)$ by carrying out high level $a b$ initio quantum chemical calculations for any polyatomic system is a challenging task. Some of the nuances involved in the process have been discussed recently by Dawes and Ndengué. ${ }^{55}$ Fitting an analytic function to the computed potential energy (PE) values or interpolating/extrapolating them numerically is an additional challenge. ${ }^{56-58}$ Briefly, the potential energy curve for a diatomic species near the potential minimum $\left(r_{\mathrm{m}}\right)$ can be approximated as that of a harmonic oscillator,

$$
V=\frac{1}{2} k x^{2}, \quad x=r-r_{\mathrm{m}} .
$$

For larger displacements, suitable analytic functions can be fitted, the simplest being the Morse potential. ${ }^{59}$ Several other analytic functions have been proposed over the years. Some of them have been reviewed elsewhere. ${ }^{56-58}$ For triatomic reactive systems, the London-Eyring-Polanyi-Sato (LEPS) formalism ${ }^{60-62}$ served as the basis for analytic fitting of the PESs in the earlier years. A diatomics-in-molecules (DIM) approach $^{63}$ was also used for some systems. As ab initio quantum chemical methods improved and the ease of computing $a b$ initio $\mathrm{PE}$ values increased and the cost of generating them came down, one realized that the LEPS and DIM formalisms did not have enough flexibility in them to fit the $a b$ initio PE values. Many body expansion methods were proposed for fitting ab initio PESs. ${ }^{64}$ That is, the PES for a triatomic system, for example, was broken into 1-, 2- and 3-body terms. While the 1-body (atomic) energy could be taken as zero (or constant), the 2-body term for each diatomic component could be reproduced by one of the analytic functions mentioned above. The 3-body term was invariably expanded in terms of a polynomial and a range function that died off at long range. Some of the earlier efforts have been described by Murrell et al. ${ }^{57}$ and Aguado and Paniagua. ${ }^{65}$ For a programme implementation of the many body approach for systems containing up to four atoms, the reader may see the recent paper by Rocha and Varandas. ${ }^{66}$ Considerable effort has been expended in recent years in fitting permutationally invariant polynomials (PIPs) to $a b$ initio data for larger (up to 11 atoms) systems. ${ }^{67-69}$ Significant progress has been made in using artificial neural networks (ANN) methods and a hybrid of the PIP and ANN methods for fitting a large number of points on the PES. ${ }^{68-71}$ The method of Gaussian Process Regression (GPR) seems like an attractive alternative for fitting a PES with a limited number of data points. ${ }^{72,73}$ Some of the earlier efforts in numerical interpolation of $a b$ initio PES points included 1D/ 2D/3D splines. ${ }^{74}$ The application of the spline-fitting approach to higher dimensions has been discussed by Patricio et al. ${ }^{75}$

In principle, if we know the coordinates and the spatial grid in which the time evolution of the wave function is to be carried out, it is a onetime effort to compute the PES on that grid of points in the coordinate space. In practice, however, one has to see which set of coordinates suits the system under investigation the best and alter the sampling of the grid as the work progresses. The concept of a potential energy curve/surface breaks down when the Born-Oppenheimer (BO) approximation breaks down. ${ }^{76-81}$ One has to deal with a number of electronic states and nonadiabatic couplings between them (see below). Double many body expansion methods have been proposed to deal with such systems. ${ }^{82}$

In what follows, we describe the three steps involved in a standard TDQMWP approach, first within the BO approximation (see Sections 2.1, 2.2 and 2.3), and then when we go beyond the BO approximation (see Section 2.4). 


\subsection{Step 1: choosing the initial wave function}

For a particle moving in the presence of a potential well or a barrier, the initial state could be represented by a minimum uncertainty Gaussian

$$
g(R)=\left(2 \pi \delta^{2}\right)^{-1 / 4} \exp \left[-\frac{\left(R-R_{0}\right)^{2}}{4 \delta^{2}}-i k_{0} R\right],
$$

where $R_{0}$ is the center of the wave packet in $R$ space. Its Fourier transform

$$
\Phi(k)=\left(\frac{2 \delta^{2}}{\pi}\right)^{1 / 4} \exp \left[-\left(k-k_{0}\right)^{2} \delta^{2}+i R_{0} k\right],
$$

corresponds to a WP centered at $k_{0}$ in $k$-space. The phase factor $\exp \left(-\mathrm{i} k_{0} R\right)$ in $g(R)$ directs the WP towards the interaction region. The average translational energy $\left\langle E_{\text {trans }}\right\rangle$ corresponds to:

$$
\left\langle E_{\text {trans }}\right\rangle=\hbar^{2}\left(k_{0}^{2}+\frac{1}{4 \delta^{2}}\right) / 2 \mu_{\mathrm{A}, \mathrm{BC}} .
$$

An optimal width parameter of $\delta=0.25-1$ a.u. of the WP is chosen in most studies. If the WP has a narrow width in momentum space, it would be spread out in position space, which would require a large spatial grid, which in turn would necessitate large computer memory and time for any dynamical study. On the other hand, if the WP is narrow in position space, it would be spread out in momentum space, compromising the energy resolution for the reaction attributes. The location of the WP is also critical in that it should be away from the interaction region and yet not too close to the outer edge of the grid to prevent reflection of the WP from the edge(s).

The initial condition $(t=0)$ for an $\mathrm{A}+\mathrm{BC}$ reactive system

$$
\mathrm{A}+\mathrm{BC}(v, j) \stackrel{E_{\text {trans }}}{\longrightarrow} \mathrm{AB}\left(v^{\prime}, j^{\prime}\right)+\mathrm{C},
$$

for example, can be defined in terms of the initial vib-rotational $(v, j)$ state of the $\mathrm{BC}$ molecule and the relative translational energy ( $\left.E_{\text {trans }}\right)$ between reactants as

$$
\Psi(r, R)=g(R) \phi_{\nu, j}(r)
$$

The translational part, $g(R)$, is usually taken as a (minimum uncertainty) Gaussian (see eqn (11) above) and $\phi_{v_{j}}(r)$ is the vib-rotational wave function of the diatomic molecule BC. To calculate reactive scattering cross sections over an energy range more accurately, the use of a sinc wave packet was proposed by Hankel et $a l .{ }^{83}$ In contrast to the Gaussian wave packet, the amplitude of the wave packet remains nearly constant over an energy range in the sinc wave packet. For photoabsorption/ dissociation processes, the wave function of the initial $(v, j)$ state of the molecule in its ground electronic state is modified using the transition dipole moment and taken as the initial wave function for dynamics on the excited state(s). For molecule-surface systems, similar suitable initial wave functions are chosen for studying the time evolution of the system.

\subsection{Step 2: time evolution of the wave function}

The evolution of a dynamical system can be followed in real time by solving the TDSE. This simple looking first-order differential equation can be solved readily if the application of the Hamiltonian operator on $\Psi$ can be computed readily. Formally,

$$
\Psi(t)=U\left(t, t_{0}\right) \Psi\left(t_{0}\right),
$$

where $U\left(t, t_{0}\right)$ is the evolution operator, which is unitary. If $\hat{H}$ is explicitly time-independent,

$$
U\left(t, t_{0}\right)=\exp \left[-\frac{i\left(t-t_{0}\right) \hat{H}}{\hbar}\right] .
$$

In the absence of an external field, $\hat{H}$ can be written as

$$
\hat{H}=\hat{T}+\hat{V}
$$

where $\hat{T}$ is the kinetic energy operator and $\hat{V}$ is the potential energy operator. Unfortunately, $\hat{T}$ and $\hat{V}$ do not commute with each other as the former is "local" in momentum space and the latter is "local" in position space. In the second-order splitoperator method, ${ }^{84}$ the evolution operator can be applied repeatedly over several slices $(\Delta t)$ through

$$
\begin{aligned}
U(\Delta t) & =\exp \left[-\frac{i \Delta t \hat{H}}{\hbar}\right] \\
& =\exp \left[-\frac{i \Delta t \hat{T}}{2 \hbar}\right] \exp \left[-\frac{i \Delta t \hat{V}}{\hbar}\right] \exp \left[-\frac{i \Delta t \hat{T}}{2 \hbar}\right] .
\end{aligned}
$$

Alternatively, the evolution operator can be approximated as

$$
U(\Delta t)=\exp \left[-\frac{i \Delta t \hat{V}}{2 \hbar}\right] \exp \left[-\frac{i \Delta t \hat{T}}{\hbar}\right] \exp \left[-\frac{i \Delta t \hat{V}}{2 \hbar}\right] .
$$

The split-operator method is norm, $\|\Psi(t)\|$, conserving. Therefore, one can easily check the robustness of the algorithm by checking the norm of the wave function. Robustness here refers to numerical stability. It also raises the question of how "long" the time evolution can be carried out numerically accurately. This is often checked by evolving the final wave function back in time and ensuring that it coincides with the wave function we started with. Tal-Ezer and Kosloff ${ }^{85}$ showed that "exact" results could be obtained using the Chebyshev polynomial method. There have been several refinements made to the split-operator approach over the years. A detailed discussion of the increased accuracy of higher order split-operator methods for a one dimensional (Morse) potential and (A + BC) reactive scattering has been given by Sun et al. ${ }^{86}$ They also compared the efficacy of the split-operator methods with the Chebyshev polynomial method. To obtain a spectral resolution of $\left( \pm 1 \mathrm{~cm}^{-1}\right)$, for example, in the dynamical attributes, the uncertainty principle requires that the time evolution be carried out for at least 5300 fs $(5.3 \mathrm{ps})$. The second order split operator method and the Chebyshev polynomial method are numerically stable and robust enough for such long time evolution. Braun et al. have shown ${ }^{87}$ that the second order split operator method was stable enough for time evolution of the order of nanoseconds and yielded eigenfunctions and eigenvalues to acceptable accuracy. Sun et al. ${ }^{86}$ have shown that for systems involving deep potential wells and/or a large number of 
narrow resonances, one may (have to) resort to higher order split operator methods.

When $\hat{H}$ is explicitly time-dependent, time ordering is important in arriving at $\Psi(t)$. Pfeifer and Levine ${ }^{88}$ and Peskin and Moiseyev ${ }^{89}$ used the $\left(t, t^{\prime}\right)$ method for this purpose. Very recently, Balanarayan and coworkers ${ }^{90}$ have proposed a $\left(t, t^{\prime}, t^{\prime \prime}\right)$ method to obtain exact results for molecules interacting with short pulse lasers. One of the bottlenecks in solving the TDSE has been in determining the operation of the kinetic energy operator on $\Psi$ for the reasons mentioned earlier. By switching to the momentum representation, the kinetic energy part can be evaluated to machine accuracy through the fast Fourier transform (FFT) route $84,91,92$

$$
\begin{aligned}
& \Psi(x)=\int \Phi(k) \mathrm{e}^{i k x} \mathrm{~d} k, \\
& \Psi^{\prime}(x)=\int \Phi(k)(i k) \mathrm{e}^{i k x} \mathrm{~d} k, \\
& \Psi^{\prime \prime}(x)=\int \Phi(k)\left(-k^{2}\right) \mathrm{e}^{i k x} \mathrm{~d} k .
\end{aligned}
$$

The second order spatial derivative of the wave function is thus obtained by Fourier transforming the wave function $\Psi(x)$, multiplying the Fourier transform $\Phi(k)$ by $\left(-k^{2}\right)$ and taking an inverse Fourier transform of the resultant product. Although this approach works well in Cartesian coordinates for a localised wave function in an extended grid, the FFT algorithm transforms a large number of zeroes into zeroes at every step of time evolution. ${ }^{93}$ A simple way to alleviate this problem is to choose a floating grid so that the FFT operation is carried out in limited space. This would save substantial computing time and memory. The difficulty with this approach is that we need to know in advance how the spread in the wave packet in $(r, R)$ space would be. Mowrey and Kouri ${ }^{94,95}$ suggested the use of an L-shaped grid to alleviate the problem. Values of the initial state selected (but summed over the final states) probability and cross section are obtained by computing the flux ( $\mathbb{F})$ across a dividing line in the product channel. ${ }^{96}$

Unfortunately, because of the finite size of the grid, part of the wave packet would get reflected from the edges. This is prevented by extending the range of the grid, which has its limitations. An alternative is to use an absorbing potential in the form of a negative imaginary potential (NIP) added to the Hamiltonian near the grid edges and this was proposed by various practitioners in the field. ${ }^{48,97-105}$ Despite the widespread use of NIPs, they were found to cause instability of various time-evolution schemes. ${ }^{106,107}$ The problem arises because the Hamiltonian becomes non-Hermitian in their presence. Mahapatra and Sathyamurthy carried out a detailed analysis and came up with a real damping function of a sine type as an alternative ${ }^{105}$

$$
f\left(X_{i}\right)=\sin \left[\frac{\pi}{2} \frac{\left(X_{\text {mask }}+\Delta X_{\text {mask }}-X_{i}\right)}{\Delta X_{\text {mask }}}\right], X_{i} \geq X_{\text {mask }}
$$

In the above equation $X_{\text {mask }}$ is the point at which the damping function is activated on the grid and $\Delta X_{\text {mask }}=X_{\text {max }}-X_{\text {mask }}$ is the width along $X$ over which the function smoothly decays from 1 to 0 with $X_{\max }$ being the maximum value of $X$ on the grid. This damping function has been used successfully in various TDQMWP studies.

The discrete variable representation (DVR) method, originally introduced in the area of molecular reaction dynamics by Light and co-workers, ${ }^{108}$ involves expanding the wave function $\Psi(x, t)$ in an orthonormal basis set $\left\{\phi_{i}(x), i=1, \ldots, N\right\}$ :

$$
\Psi(x, t)=\sum_{i=1}^{N} a_{i}(t) \phi_{i}(x)
$$

where $a_{i}(t)=\int \phi_{i}^{*}(x) \Psi(x, t) \mathrm{d} x$.

It uses a quadrature rule, consisting of a set of quadrature points $\left\{x_{i}, i=1, \ldots, N\right\}$ and the corresponding weights $\left\{w_{i}\right.$, $i=1, \ldots, N\}$ such that

$$
a_{i}(t)=\sum_{j=1}^{N} w_{j} \phi_{i}^{*}\left(x_{j}\right) \Psi\left(x_{j}, t\right) .
$$

As a result,

$$
\Psi(x, t)=\sum_{i=1}^{n} \sum_{j=1}^{N} w_{j} \phi_{i}^{*}\left(x_{j}\right) \Psi\left(x_{j}, t\right) \phi_{i}(x)=\sum_{j=1}^{N} \Psi_{j} \psi_{j}(x) .
$$

The functions

$$
\psi_{j}(x)=\sqrt{w_{j}} \sum \phi_{i}^{*}\left(x_{j}\right) \phi_{i}(x)
$$

form a set of orthonormal basis functions in the discrete representation and

$$
\Psi_{j}=\sqrt{w_{j}} \Psi\left(x_{j}, t\right)
$$

is the amplitude of the wave function on the $j$ th basis function. The $n$th order spatial derivative of the wave function is then obtained from

$$
\frac{\partial^{n} \Psi(x, t)}{\partial x^{n}}=\sum_{j=1}^{N} \Psi_{j} \frac{\partial^{n} \psi_{j}(x)}{\partial x^{n}}
$$

where

$$
\frac{\partial^{n} \psi_{j}(x)}{\partial x^{n}}=\sqrt{w_{j}} \sum_{i=1}^{N} \phi_{i}^{*}\left(x_{j}\right) \frac{\partial^{n} \phi_{i}(x)}{\partial x^{n}},
$$

which can be computed at all $x_{j}, j=1, \ldots, N$, once at the beginning of the computations and retrieved subsequently from storage during the propagation.

Although the DVR method scales as $N^{2}$ when compared to the FFT method, which scales as $N \ln N$, the former has the distinct advantage in that there is no need to transform the wave function to momentum space and back at every timestep. It is also possible to keep the number of basis functions required in the expansion to a minimum if an appropriate basis set is chosen keeping in mind the nature of the potential.

\subsection{Step 3: analysing the time evolved wave function}

2.3.1 Bound state problem. Although the bound states of various systems such as the particle-in-a-box, harmonic oscillator, hydrogen atom, etc., were obtained by Schrödinger by solving the TISE, the same can be obtained via the time-dependent 
route by choosing a suitably located initial wave function, time evolving it, computing the autocorrelation function $C(t)$ and Fourier transforming $C(t)$ to obtain the power spectrum $I(E)$ over the bound state eigenvalues $E_{n}$ and projecting the corresponding eigenfunctions $\Psi\left(E_{n}\right)$ from the wave function at different time intervals. ${ }^{31,109}$

$$
\begin{gathered}
C(t)=\langle\Psi(0) \mid \Psi(t)\rangle, \\
I(E)=\left|\int_{-\infty}^{\infty} C(t) \mathrm{e}^{i E t / \hbar} \mathrm{d} t\right|^{2}, \\
\Psi\left(E_{\mathrm{n}}\right)=\int_{-\infty}^{\infty} \Psi(t) \mathrm{e}^{i E_{\mathrm{n}} t / \hbar} \mathrm{d} t .
\end{gathered}
$$

The Fourier transform involves time in the range $[-\infty, \infty]$. In reality, the time evolution is carried out for a finite time $T$. Using time reversal symmetry, the integral can be written as if the time evolution were carried out for $2 T .{ }^{110}$ This helps in improving the energy resolution of the eigenstates as the length of the time evolution is related to the energy resolution through the uncertainty principle. Often one uses a window function $w(t)$ to account for the finite value of $T$ and to ensure that spurious eigenvalues are not obtained.

$$
I(E)=\left|\frac{1}{T} \int_{0}^{T} C(t) w(t) \mathrm{e}^{i E t / \hbar} \mathrm{d} t\right|^{2}
$$

and

$$
\Psi\left(E_{n}\right)=\frac{1}{T} \int_{0}^{T} \Psi(t) \mathrm{e}^{i E_{\mathrm{n}} t / \hbar} \mathrm{d} t
$$

It is common to use a (Hanning) window function: ${ }^{111}$

$$
w(t)= \begin{cases}1-\cos \left(\frac{2 \pi t}{T}\right), & \text { if } 0 \leq t \leq T \\ 0, & \text { if } t>T\end{cases}
$$

This approach reveals the quasi-bound states and hence the transition state resonances as well. The size and the range of the grid are often extended and the time evolution repeated to ensure that the results obtained are converged with respect to the (finite) range of the grid used.

2.3.2 Photo-excitation problem. Photo-absorption cross sections and photodissociation cross sections can also be computed by computing $C(t)$. In this case, the initial WP $\left(\Phi_{\mathrm{i}}(0)\right)$ is prepared by promoting the initial wave function $\Psi_{\mathrm{i}}(0)$ in the ground electronic state, for example, with the help of the excitation operator $\vec{\mu} \cdot \vec{E}$ :

$$
\Phi_{\mathrm{i}}(0)=\vec{\mu} \cdot \vec{E} \Psi_{\mathrm{i}}(0)
$$

where $\vec{\mu}$ is the transition dipole vector and $E$ the electric field of the photon. The time evolution of the promoted state is governed by the Hamiltonian $\left(\hat{H}_{\mathrm{ex}}\right)$ of the excited state $(\mathrm{s})$ and

$$
\Phi_{\mathrm{i}}(t)=\exp \left[-\frac{i t \hat{H}_{\mathrm{ex}}}{\hbar}\right] \Phi_{\mathrm{i}}(0) .
$$

The autocorrelation function $C_{\mathrm{ii}}(t)$ would then be

$$
C_{\mathrm{ii}}(t)=\left\langle\Phi_{\mathrm{i}}(0) \mid \Phi_{\mathrm{i}}(t)\right\rangle .
$$

The absorption spectrum $\sigma_{\mathrm{A}}(\omega)$ would be:

$$
\sigma_{\mathrm{A}}(\omega)=\frac{2 \pi \omega}{3 \hbar c} \int_{-\infty}^{\infty} C_{\mathrm{ii}}(t) \exp \left[-\frac{i t E}{\hbar}\right] \mathrm{d} t
$$

where $\omega$ is the frequency of the incident radiation and $E=E_{\mathrm{g}}+$ $\hbar \omega$ with $E_{\mathrm{g}}$ the internal energy of the molecule in its ground electronic state. The emission spectrum from an excited state can be obtained by reversing the role of the initial and final electronic states. The cross correlation function $C_{\mathrm{fi}}(t)$, also called the Raman correlation function, is obtained from

$$
C_{\mathrm{fi}}(t)=\left\langle\Phi_{\mathrm{f}} \mid \Phi_{\mathrm{i}}(t)\right\rangle,
$$

where $\Phi_{\mathrm{f}}=\vec{\mu} \cdot \vec{E} \Psi_{\mathrm{f}}$ is the wave function corresponding to the (final) vibrational state $\mathrm{f}$ in the ground electronic state (see Fig. 2 for an illustration). Raman amplitude $\alpha_{\mathrm{fi}}(\omega)$ is obtained from

$$
\alpha_{\mathrm{fi}}(\omega)=\frac{i}{\hbar} \int_{0}^{\infty} C_{\mathrm{fi}}(t) \mathrm{e}^{i\left(\omega+\omega_{\mathrm{i}}\right) t} \mathrm{~d} t .
$$

The quantity $\omega_{\mathrm{i}}$ in eqn (40) is the frequency of the incident radiation. The "observable" Raman scattering intensity or the Raman excitation profile $I_{\mathrm{fi}}(\omega)$ follows:

$$
I_{\mathrm{fi}}(\omega) \propto \omega \omega_{\mathrm{s}}^{3}\left|\alpha_{\mathrm{fi}}(\omega)\right|^{2},
$$

where $\omega_{\mathrm{s}}$ is the frequency of the scattered radiation. An account of the Raman excitation profile can be found elsewhere. ${ }^{112}$

The utility of the above mentioned methodology in studying photo-excitation processes involving one or more excited states in one dimension has been demonstrated by Mahapatra et al. ${ }^{31}$ For a standard treatment of the subject, the reader may refer to ref. 24 and 113 .

2.3.3 Scattering problem. In the case of elastic/inelastic/ reactive scattering, state-to-state transition/reaction probabilities can be computed by projecting the time evolving wave function onto the corresponding final states. ${ }^{38}$ A sum over the final states and $l$-averaging ( $l$ is the orbital angular momentum quantum number) would yield initial state-selected integral cross section (ICS) values. Appropriate averaging over the initial vib-rotational states and $E_{\text {trans }}$ of the reactants would yield

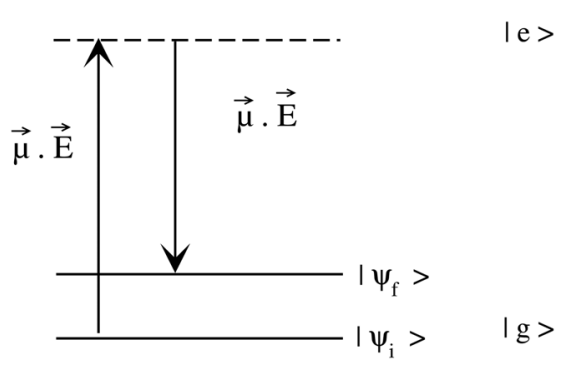

Fig. 2 The ground and the first excited electronic states are represented by $|g\rangle$ and $|e\rangle$, respectively. The wave function for the initial and final vibrational state (for Stokes shift) in the ground electronic state is represented by $\left|\psi_{\mathrm{i}}\right\rangle$ and $\left|\psi_{\mathrm{f}}\right\rangle$, respectively. 
thermal rate coefficients, when needed. It is possible to extract state-to-state differential cross sections (DCSs) as well. ${ }^{114}$ It is possible to investigate the underlying resonances also. ${ }^{16}$ The initial state-selected (product state not specified; summed over all energetically allowed product states) reaction probability is computed by computing the (cumulative) flux ${ }^{115}$

$$
\mathbb{F}(R, r, t)=\frac{\hbar}{\mu^{\prime}} \operatorname{Im}\left[\Psi^{*}(R, r, t) \nabla \Psi(R, r, t)\right]
$$

across a dividing surface in the product channel. This method has been used successfully to determine the cross section for exchange as well as collision induced dissociation processes (see for example ref. 116). A wave function splitting algorithm ${ }^{117}$ has been used to obtain final state-resolved reaction probabilities and hence reaction cross sections. The difficulties involved in using the rearrangement channel dependent Jacobi coordinates are circumvented by a reactant-product-decoupling (RPD) scheme. ${ }^{118}$ An alternative is to use a product coordinate based (PCB) scheme. ${ }^{119-121}$ One such scheme that turned out to be promising computationally was the real wave packet (RWP) method of Gray and Balint-Kurti. ${ }^{122}$ The RWP method relies on a one-to-one functional mapping ( $\cos ^{-1}$ mapping) of the Hamiltonian which leads to a Chebyshev polynomial based iterative equation for the time propagation of the WP. This scheme requires the imaginary component of the WP only once to start the iterations and the rest of the calculations require only the real part of the WP and hence the method is computationally faster. Letting $\Psi=q+i p$, the iterative equation for the time propagation of the WP in this method reads ${ }^{122}$

$q^{J K^{\prime}}\left(R^{\prime}, r^{\prime}, \gamma^{\prime}, t+\tau\right)=-q^{J K^{\prime}}\left(R^{\prime}, r^{\prime}, \gamma^{\prime}, t-\tau\right)+2 H^{\prime} q^{J K^{\prime}}\left(R^{\prime}, r^{\prime}, \gamma^{\prime}, t\right)$.

In the above equation $q^{J K^{\prime}}$ is the real part of the initial WP that is defined in the reagent Jacobi frame (as above) and transformed to the product Jacobi frame (the primed quantities) prior to propagation. The quantity $K^{\prime}$ is analogous to $K$, defined in the product Jacobi frame. The scaled Hamiltonian in the product Jacobi coordinate is denoted by $\hat{H}^{\prime}{ }^{121}$ To start the iteration eqn (43) requires $q^{J K^{\prime}}$ at the first time step $\tau$. The imaginary part of the WP is utilized once for this purpose and is evaluated by

$$
\begin{aligned}
q^{J K^{\prime}}\left(R^{\prime}, r^{\prime}, \gamma^{\prime}, \tau\right)= & \hat{H}^{\prime} q^{J K^{\prime}}\left(R^{\prime}, r^{\prime}, \gamma^{\prime}, 0\right) \\
& -\sqrt{1-\hat{H}^{\prime} p^{J K^{\prime}}\left(R^{\prime}, r^{\prime}, \gamma^{\prime}, 0\right) .}
\end{aligned}
$$

The action of the operator $\sqrt{1-\hat{H}^{\prime}}$ on $p^{J K^{\prime}}$ in eqn (44) is carried out by expanding it in terms of Chebyshev polynomials of the first kind. ${ }^{37}$ In contrast to the flux operator method in the reagent Jacobi coordinates, the analysis of the product states is done by projecting the time evolved WP on the vib-rotational wave function of the product on an analysis line in the product channel. The resulting time-dependent coefficients are Fourier transformed to the energy domain and finally the energy dependent scattering matrix elements are derived. This scheme was quite successful in calculating the reaction cross section for scattering systems of varying complexity. ${ }^{121,123,124}$ The salient features of the WP propagation within the TDQM framework on a grid in the reagent or product Jacobi coordinates have been reviewed by Balint-Kurti. ${ }^{37}$ Various approximate schemes introduced in the literature from time-to-time to deal with photodissociation and reactive scattering problems were also discussed by Balint-Kurti. ${ }^{37}$

Yet another approach is to use a reactant coordinate based (RCB) scheme. ${ }^{125,126}$ As mentioned earlier, one way to avoid the channel-dependent Jacobi coordinates is to work in hyperspherical coordinates. The number of papers published using hyperspherical coordinates in the TDSE framework is rather limited ${ }^{127-131}$ because of the difficulty in the final state analysis. Another practical aspect of the TDQMWP approach (applicable to the TIQM approach as well) is the number of $J$ values for which the calculations have to be repeated. It is also known that with an increase in the value of $J$, the initial wave packet has to be located farther out in the reactant channel because of the centrifugal barrier. This means that the grid has to be extended farther for calculations for large $J$. Understandably, some of the earlier 3D calculations were carried out for $J=0$ only. With an increase in the computational resources becoming available, calculations were carried out for larger $J$ until the results converged with respect to $J$, but using the centrifugal sudden approximation. Later calculations included Coriolis coupling, but were limited to small helicity $(K)$ numbers. ${ }^{9,132-135}$ Parallel computing resources have enabled the inclusion of large $J$ and $K$ numbers. ${ }^{52,136-138}$ Goldfield and Gray ${ }^{52}$ devised an algorithm for efficient parallelization of $K$ substates. Later it was employed to calculate the ICS for the $\mathrm{H}+\mathrm{O}_{2}$ reactive system by Meijer and Goldfield. ${ }^{139}$ In recent years, the RWP method has been used to carry out large scale converged parallel calculations. ${ }^{123,124}$ However, there has been no report in the literature of results that are both $J$ - and $K$-converged so far. The distinct advantage of the TDQMWP approach is that the reaction attributes over a range of $E_{\text {trans }}$ can be computed in one go, in contrast to the TIQM approach, which requires the massive calculations to be repeated for each $E_{\text {trans }}$ value that we are interested in. Unfortunately, the TDQMWP method is not suited for investigations at low energies as it would require a larger spread in the wave packet in the coordinate space and would be relatively time consuming. The TIQM method, on the other hand, has an advantage in that the number of vibrotational channels to be included in the dynamics would be limited at low $E_{\text {trans }}$.

\subsection{Adiabatic to nonadiabatic via the diabatic route}

The vast majority of early developments of the TDQMWP methodology were restricted to chemical processes occurring on a single adiabatic PES. Increased realization of the importance of vibronic coupling in electronic spectroscopy and photo-dissociation processes led to a treatment of reactive chemical processes on coupled multi-sheet PESs by the TDQMWP technique in subsequent years.

Historically, the seminal paper of von Neumann and Wigner $^{140}$ laid the foundation for the study of nonadiabatic interactions in 1929 and the importance of the crossing of PESs was recognized in the 1930 s. $^{141-143}$ Nearly two decades later, 
pioneering contributions by Herzberg and Longuet-Higgins ${ }^{144-146}$ led to the development of new concepts in the area. The field underwent monumental growth thereafter and a variety of molecular processes have been studied by several groups $^{77,147-160}$ over the years. A review of all the developments on PES crossing is beyond the scope of the present perspective. Therefore, we focus on the essential aspects of treating the nuclear dynamics on coupled electronic states by the TDQMWP methodology here.

The immediate and the most fundamental consequence of crossing of PESs is a break-down of the Born-Oppenheimer (BO) approximation. ${ }^{161,162}$ The electronic and nuclear motions get entangled and they occur concurrently. In the framework of the Born-Oppenheimer-Huang formalism, ${ }^{161,162}$ the nuclear Schrödinger equation in its time-independent form reads

$$
\left[T_{n}^{j}(\mathbf{R})+V^{j}(\mathbf{R})-E^{j}\right] \Psi_{n}^{j}(\mathbf{R})=\sum_{i=1}^{\infty} \Lambda_{j i}(\mathbf{R}) \Psi_{n}^{i}(\mathbf{R})
$$

In the above eqn (45), $n$ is the nuclear index. The quantity $T_{n}^{j}$ $(\mathbf{R})$ is the nuclear kinetic energy operator of the $j$ th electronic state depending on the set of nuclear coordinates $\mathbf{R}$. The adiabatic potential energy of the $j$ th electronic state resulting from the solution of the electronic Schrödinger equation,

$$
H_{\mathrm{el}}^{j}(r ; \mathbf{R}) \psi_{\mathrm{el}}^{j}(r ; \mathbf{R})=V^{j}(\mathbf{R}) \psi_{\mathrm{el}}^{j}(r ; \mathbf{R})
$$

within the BO approximation is given by $V^{j}(\mathbf{R})$. The electronic Hamiltonian of the $j$ th electronic state including inter-nuclear repulsion is denoted by $H_{\mathrm{el}}^{j}(\mathbf{r} ; \mathbf{R})$ and it depends on the set of electronic coordinates $\mathbf{r}$ explicitly and parametrically on $\mathbf{R}$. The quantity, $E^{j}$, represents the total molecular energy of the $j$ th electronic state. $\Psi_{n}^{j}(\mathbf{R})$ is the nuclear wave function in the $j$ th electronic state. On the right hand side of eqn (45), the summation runs over the complete set of electronic states. In principle, the number of states is infinity, but, in practice, it is restricted to a finite number. This issue is addressed later in the text. $\Lambda_{j i}(\mathbf{R})$ represents the element of the nonadiabatic coupling operator (say $\boldsymbol{\Lambda}$ ) and is given by ${ }^{151}$

$$
\Lambda_{j i}(\mathbf{R})=-\sum_{n} \frac{\hbar^{2}}{M_{n}} F_{j i} \frac{\partial}{\partial R_{n}}-\sum_{n} \frac{\hbar^{2}}{2 M_{n}} G_{j i},
$$

where $M_{n}$ is the mass of the $n$th nucleus and

$$
\begin{aligned}
F_{j i}(\mathbf{R}) & =\left\langle\psi_{\mathrm{el}}^{j}(r ; \mathbf{R})\left|\nabla_{n}\right| \psi_{\mathrm{el}}^{i}(\mathbf{r} ; \mathbf{R})\right\rangle, \\
G_{j i}(\mathbf{R}) & =\left\langle\psi_{\mathrm{el}}^{j}(r ; \mathbf{R})\left|\nabla_{n}{ }^{2}\right| \psi_{\mathrm{el}}^{i}(\mathbf{r} ; \mathbf{R})\right\rangle,
\end{aligned}
$$

with $\nabla_{n} \equiv \frac{\partial}{\partial R_{n}}$. It is obvious from eqn (48a) and (48b) that when $F_{j i}$ and $G_{j i}$ are non-zero, the electronic states $i$ and $j$ are coupled through nuclear motion. This dynamical coupling $\left(\Lambda_{j i}\right)$ is zero in the BO approximation. When the adiabatic potential energy, $V^{j}(R)$, is corrected by retaining the diagonal elements of the scalar coupling $\left(G_{j i}\right)$, it is known as the Born-Huang (BH) correction. ${ }^{162}$ Using the Hellmann-Feynman theorem, ${ }^{163}$ the elements of the vector (derivative) coupling operator ( $c f$, eqn (48a)) are given by ${ }^{148,151}$

$$
F_{j i}(\mathbf{R})=\frac{\left\langle\psi_{\mathrm{el}}^{j}(\mathbf{r} ; \mathbf{R})\left|\nabla_{n} H_{\mathrm{e}}(\mathbf{r} ; \mathbf{R})\right| \psi_{\mathrm{el}}^{i}(\mathbf{r} ; \mathbf{R})\right\rangle}{V^{j}(\mathbf{R})-V^{i}(\mathbf{R})} .
$$

At the point of degeneracy of electronic states, $V^{j}(\mathbf{R})=V^{i}(\mathbf{R})$, and $F_{j i}(\mathbf{R})$ becomes singular. Since the nonadiabatic coupling operator is inversely proportional to the nuclear mass ( $c f$. eqn (47)), for widely separated electronic states the mass factor ensures that $\boldsymbol{\Lambda}$ is negligible and the BO approximation is justified. However, if the electronic states are closely spaced in energy, the denominator of eqn (49) outweighs the mass factor, leading to a break-down of the BO approximation.

The electronic representation discussed above is referred to as the adiabatic representation and the coupling between states is defined by the off-diagonal elements of the nuclear kinetic energy operator, known as nonadiabatic coupling terms (NACTs) or nonadiabatic coupling matrix elements (NACMEs). Furthermore, the participating electronic states in this representation lack analytic continuation owing to the diverging derivative of the electronic wave function. ${ }^{148}$ Therefore, the adiabatic representation is ill-suited for a numerical solution of the Schrödinger equation ( $c f$. eqn (1)) when the nonadiabatic coupling becomes important.

To circumvent the limitations of the adiabatic representation, one resorts to a diabatic representation introduced by Litchen ${ }^{164}$ in the context of ion-atom collisions. Diabatic electronic states were defined by Smith ${ }^{165}$ in terms of differential equations of the adiabatic-to-diabatic transformation (ADT) matrix requiring the singular derivative coupling of the adiabatic basis to vanish in this representation. This work focused on atom-atom collisions and Baer and coworkers ${ }^{166,167}$ extended it to atom-diatom collisions. Mathematically, diabatic electronic states are obtained by an orthogonal transformation of a subset of the complete set of adiabatic states as

$$
\begin{gathered}
\Psi^{\mathrm{d}}=\mathbf{S} \Psi^{\mathrm{ad}}, \\
H^{\mathrm{d}}=\mathbf{S} H^{\mathrm{ad}} \mathbf{S}^{\dagger}=T_{n} \mathbf{1}+\mathbf{U} .
\end{gathered}
$$

The superscripts $\mathrm{d}$ and ad refer to the diabatic and adiabatic representations, respectively, and $\mathbf{S}$ defines the ADT matrix. ${ }^{165}$ For a two state problem, the ADT matrix is given by

$$
\mathbf{S}=\left(\begin{array}{cc}
\cos \theta(\mathbf{R}) & \sin \theta(\mathbf{R}) \\
-\sin \theta(\mathbf{R}) & \cos \theta(\mathbf{R})
\end{array}\right)
$$

The ADT angle $\theta(\mathbf{R})$ depends on the set of nuclear coordinates R. Eqn (50b) above reiterates that the nuclear kinetic energy operator is diagonal ( $\mathbf{1}$ is a $2 \times 2$ diagonal unit matrix) and the electronic potential energy ( $\mathbf{U})$ contains off-diagonal elements in the diabatic representation. It is important to note that diabatic electronic states are not eigenstates of the electronic Hamiltonian ( $c f$. eqn (46)) and are not "observables". However, the analytic continuation of the electronic energy is restored in this representation (as the diverging derivative coupling is removed). As a result, the diabatic representation 
is amenable to numerical application. The requirement that $F_{j i}$ $(j \neq i)=0$ in eqn (48a) in this new basis leads to the following differential equation of the ADT angle ${ }^{166}$

$$
\nabla \theta(\mathbf{R})+F_{j i}=0 .
$$

For a one dimensional problem (a diatomic system), the above equation can be solved trivially for $\theta(\mathbf{R})$ with the aid of $a b$ initio computed NACTs, $F_{j i}{ }^{168}$ For a multi-dimensional problem, it was proposed to carry out the integration in a stepwise fashion along each coordinate. The fact that the final result is invariant to the order of such sequential operations requires that $\nabla \times \mathbf{F}=0$ ( $\mathbf{F}$ being the derivative coupling matrix), known as the curl condition. ${ }^{166}$ The summation on the right hand side of eqn (45) runs over the complete set of adiabatic electronic basis states. The curl condition is satisfied only when this set is complete. The situation is trivial in diatomic systems for which there is only one coordinate and there is no curl condition. However, for polyatomic systems, construction of diabatic electronic states by satisfying the curl condition is not possible when a small subset of interacting electronic states is considered for the convenience of numerical computation. ${ }^{169}$

The above mentioned difficulties led colleagues to develop several approximate schemes to construct diabatic electronic states. Readers are referred to several review articles that have appeared over the years ${ }^{170-172}$ for a comprehensive account of the subject. We mention here only one such scheme introduced by Thiel and Köppel ${ }^{173}$ for an $(E \otimes e)$-Jahn-Teller (JT) system and later extended to a more general case introducing the concept of regularized diabatic states. ${ }^{174}$ In these schemes, the diabatic states are constructed from the adiabatic potential energies by circumventing the tedious calculations of NACTs. The important aspect of the removability of the leading derivative coupling terms in the neighborhood of the intersection seam is ensured in these schemes through numerical tests and practical applications. ${ }^{173,174}$ The results presented later for the $\mathrm{H}+\mathrm{H}_{2}$ reactive system in this perspective were obtained by utilizing this scheme of diabatization. It is worth noting here that the cusp of the potential energy curves in one dimension as defined by the singular derivative coupling evolves into conical intersections (CIs) of PESs in higher dimensions. The surfaces remain degenerate along a hypersurface in $n-2$ dimensional ( $n$ is the number of nuclear degrees of freedom) space and the locus of the degeneracy defines the seam of the CIs. CIs of PESs are known to be ubiquitous and they open up a variety of new mechanistic pathways in the dynamics of polyatomic molecular systems. ${ }^{77,145,149,151,157-160,175,176}$

The second major consequence of potential energy curve crossings due to CIs is that the adiabatic electronic wave function ceases to be single-valued. It was shown by Herzberg and Longuet-Higgins ${ }^{145}$ that a real adiabatic electronic wave function changes sign when it traverses around a CI in a closed loop an odd number of times. To restore the single-valued nature of the wave function, Longuet-Higgins and coworkers ${ }^{14,146}$ introduced an additional phase factor to the wave function with the requirement that it also changes sign when transported along the closed loop around a CI. Such a geometry dependent phase factor is known as a Longuet-Higgins phase or geometric phase ${ }^{146,177}$ or in a more general context a Berry phase ${ }^{178}$ in the literature. It is important to note here that prior to the work of Longuet-Higgins (and Berry) such a phase change was originally discovered by Pancharatnam in crystal optics. ${ }^{179}$

Subsequent to the work of Longuet-Higgins and coworkers, ${ }^{144,146}$ Mead and Truhlar ${ }^{150}$ considered two possibilities: (1) to impose a multi-valued boundary condition on the nuclear wave function and (2) to multiply the nuclear wave function by an extra phase factor, which changes sign upon an excursion around a CI in a closed loop. Both the possibilities have been examined rigorously by various practitioners in the field. Possibility (1) is well-studied in a TIQM framework and is cumbersome to implement as a basis set expansion of the nuclear wave function on a grid in a TDQMWP study. Possibility (2) is easily implementable in the TDQMWP framework. When possibility (2) is considered, the nuclear Schrödinger equation acquires a vector potential. ${ }^{150}$ This topological phase change has been discussed by many researchers in different contexts (see the review article in ref. 180). For a two-state system, Baer ${ }^{181}$ showed that the Longuet-Higgins phase ${ }^{144,146}$ is not arbitrary and is given by a first-order differential equation identical to the one satisfied by the ADT angle

$$
\theta\left(s_{1}\right)=\theta\left(s_{0}\right)-\int_{s_{0}}^{s_{1}} \mathrm{~d} s F_{12},
$$

where $s_{0}$ and $s_{1}$ are two points on a path $F$ in the configuration space. A unique solution of the above equation results when curl $F_{12}=0$. It can also be seen that the above equation is reminiscent of the equation for the Berry phase $(\gamma)$ for adiabatic evolution of a quantum system along a closed loop ${ }^{182}$

$$
\gamma=-\oint_{c} F_{12} \mathrm{~d} s
$$

It is evident that the nonadiabatic coupling operator $\boldsymbol{\Lambda}$ contains contributions from the derivative coupling, $\mathrm{BH}$ correction and the geometric phase (GP). In a coupled electronic state dynamics study, it is necessary to include all three factors in the TDQMWP formalism discussed above for the single surface case. Since the adiabatic electronic representation is not suitable for numerical applications, the TDSE is numerically solved in a diabatic representation. However, it is more realistic to prepare the initial state in the adiabatic representation and to carry out the propagation in the diabatic representation. The final analysis can be done in either of the two representations. The time evolution schemes, viz., split-operator scheme, Chebyshev polynomial method, etc. are extended to the coupled state representation. For further numerical and technical details of the solution of the TDSE in a coupled diabatic representation, readers are referred to ref. 183. In a reaction dynamics study the quantum flux operator can be represented in a diabatic as well as in the adiabatic electronic representation. ${ }^{184,185}$ Since the kinetic energy operator is diagonal in a diabatic basis, the flux operator also has the same property. The total reactive flux in this representation is the sum of the reactive flux components 
calculated at the product asymptote of each diabatic state. The flux operator, on the other hand, contains off-diagonal elements as the kinetic energy operator is non-diagonal in the adiabatic representation. The off-diagonal elements of the flux operator can make contributions when the product channel in each coupled electronic state is open at a given energy. It is, however, found that the reaction probability values calculated in either way are identical in magnitude. ${ }^{184,185}$ The application of the above formalism to study the nuclear dynamics in triatomic hydrogen is discussed further below.

\section{Applications}

\subsection{Bound states of molecular species}

Although the bound and the quasi-bound states of molecular species could be determined by solving the TISE, one could solve the TDSE and obtain the same results with comparable accuracy. In addition, one could identify the nature of resonances, if any, and estimate their lifetimes too.

Perhaps the first demonstration of the utility of the TDQMWP method in computing the bound states for a molecular system was for a 1D double well potential and 2D HenonHeiles potential. ${ }^{84}$ That was followed by the computation of bound states for $\mathrm{SO}_{2}, \mathrm{O}_{3}$ and $\mathrm{H}_{2} \mathrm{O} .{ }^{91}$

Maiti et al. ${ }^{186}$ found only one bound state for $\mathrm{HeH}_{2}{ }^{+}$on the McLaughlin-Thompson-Joseph-Sathyamurthy (MTJS) PES, but one set of two peaks for $\mathrm{HeHD}^{+}$and another set for $\mathrm{HeDH}^{+}$, in three dimensions, for $J=0$ using the TDQMWP approach. Interestingly, TDQMWP calculations ${ }^{187}$ on the Palmieri et al. PES revealed several bound states for $\mathrm{HeH}_{2}{ }^{+}$in three dimensions for $J=0$. In addition, their studies revealed a number of quasibound states that could be identified as shape resonances or Feshbach resonances (see above). They could also determine the eigenfunctions of several quasibound states and investigate their characteristics in terms of hyperspherical modes and periodic orbits. ${ }^{188}$ Recently, Koner et al. ${ }^{189}$ have determined the bound states of $\mathrm{HeH}_{2}{ }^{+}$for $J=0$ as well as $J>0$ using their newly constructed PES.

Panda and Sathyamurthy ${ }^{190}$ reported the bound states of $\mathrm{He}_{2} \mathrm{H}^{+}$and $\mathrm{He}_{2} \mathrm{D}^{+}$in three dimensions on a newly computed $a b$ initio PES. They found a slightly larger number of bound states than what was reported by Lee and Secrest earlier. ${ }^{191}$ Very recently, Koner et al. ${ }^{192}$ have computed the bound states of $\mathrm{N}_{3}{ }^{+}$ in three dimensions, for $J=0$, using the TDQMWP method on a newly computed $a b$ initio PES and found that the resulting lowest 20 bound states were in excellent agreement with those obtained using the TIQM method and DVR3D code available. ${ }^{193}$

\subsection{Reactive scattering on a single (adiabatic) PES}

A detailed dynamical theory would predict the state-to-state differential cross section at the molecular level and rate coefficients at the macro level and other observables at an intermediate level for a chemical reaction. The first TDQMWP calculation $^{5}$ in the year 1959 predicted the rate coefficient for a model collinear $\left(\mathrm{H}, \mathrm{H}_{2}\right)$ exchange reaction. Subsequent
TDQMWP studies have been able to predict the reaction probabilities in two and three dimensions and differential and integral cross sections for the reaction

$$
\mathrm{H}+\mathrm{H}_{2} \rightarrow \mathrm{H}_{2}+\mathrm{H}
$$

(in three dimensions) for different initial $(v, j)$ and final $\left(v^{\prime}, j^{\prime}\right)$ vibrotational levels of the reactant and product molecules, respectively, on the ground electronic potential energy surface. For a review of the dynamics of exchange reaction (R1), the reader may refer to ref. 21 and 194 .

The reaction

$$
\mathrm{H}^{-}+\mathrm{H}_{2} \rightarrow \mathrm{H}_{2}+\mathrm{H}^{-}
$$

resembles the neutral analog in terms of the location of the transition state and has a barrier height of $0.4648 \mathrm{eV}$, only slightly higher than $0.4249 \mathrm{eV}$ for reaction (R1) in the collinear geometry. The time evolution of a wave packet on the StärckMeyer (SM) ${ }^{195}$ ab initio PES for the collinear exchange reaction (R2) revealed a transition state spectrum rich in dynamical resonances. ${ }^{196}$ Plots of the computed reaction probability $\left(P^{\mathrm{R}}\right)$ values as a function of $E_{\text {trans }}$ revealed a large number of oscillations in the curve for the exchange reaction (R2) when compared to the limited number of oscillations corresponding to the opening up of different vibrational channels for the product molecule in the $P^{\mathrm{R}}\left(E_{\text {trans }}\right)$ curve for (R1). ${ }^{197}$

A new $a b$ initio PES was generated for reaction (R2) in three dimensions and fitted to an analytic function by Panda and Sathyamurthy. ${ }^{198}$ TDQMWP studies using an L-shaped grid for $J=0$ showed minor oscillations in the $P^{\mathrm{R}}\left(E_{\text {trans }}\right)$ curve for $v=0$ on the Panda-Sathyamurthy PES and were superimposable on the results obtained using the TIQM method and the ABC code published by Skouteris et al. ${ }^{199}$ The number and magnitude of oscillations in the $P^{\mathrm{R}}\left(E_{\text {trans }}\right)$ curve increased with an increase in $v$ from 0 to 1 and 2 and decreased in going from $j=0$ to 1 to 2 . Our results were comparable to the results of Jaquet and Heinen $^{200}$ on the SM surface and differed significantly from the results of Mahapatra ${ }^{201}$ on the DIM surface. Understandably, the latter surface is semi-empirical whereas the SM surface is $a b$ initio.

Panda et al. ${ }^{202}$ extended their studies by including $J$ values up to 40 and 60 for $\left(\mathrm{H}^{-}, \mathrm{H}_{2}\right)$ collisions and isotopic variants $\left(\mathrm{H}^{-}, \mathrm{D}_{2}\right)$ and $\left(\mathrm{D}^{-}, \mathrm{H}_{2}\right)$ and computed integral reaction cross section values. The results for $\left(\mathrm{H}^{-}, \mathrm{D}_{2}\right)$ were comparable to the experimental results of Zimmer and Linder ${ }^{203}$ for $E_{\text {trans }} \leq 1.5 \mathrm{eV}$ and those of Huq et $a .^{204}$ at $3.0 \mathrm{eV}$. Our results for both $\left(\mathrm{H}^{-}, \mathrm{D}_{2}\right)$ and $\left(\mathrm{D}^{-}, \mathrm{H}_{2}\right)$ were significantly larger than the more recent experimental results of Haufler et $a .^{205}$ Giri and Sathyamurthy ${ }^{206}$ examined the influence of $j$ on the integral reaction cross section for both $\left(\mathrm{H}^{-}, \mathrm{D}_{2}(v=0)\right)$ and $\left(\mathrm{D}^{-}, \mathrm{H}_{2}(v=0)\right)$ collisions, within the centrifugal sudden approximation, and found that the $j$-weighted integral reaction cross section values were in good agreement with the experimental results of Zimmer and Linder for $E_{\text {trans }}$ up to $1.5 \mathrm{eV}$ and with the results reported by Haufler et al. for $E_{\text {trans }}<1.0 \mathrm{eV}$. It is worth pointing out that the experimental results of Haufler et al. showed a lower reaction threshold than what was predicted by theory. 
Our group has investigated the dynamics of the exchange reaction

$$
\mathrm{He}+\mathrm{H}_{2}^{+} \rightarrow \mathrm{HeH}^{+}+\mathrm{H}
$$

and its isotopic variants

$$
\mathrm{He}+\mathrm{HD}^{+} \rightarrow \mathrm{HeH}^{+}+\mathrm{D} ; \mathrm{HeD}^{+}+\mathrm{H}
$$

using the TDQMWP approach over the years. We demonstrated the utility of the method by computing the reaction cross section for the collision induced dissociation process

$$
\mathrm{He}+\mathrm{H}_{2}{ }^{+} \rightarrow \mathrm{He}+\mathrm{H}+\mathrm{H}^{+}
$$

as well in recent years. We provide an overview of how different levels of theory were used to investigate the dynamics of reactions (R3), (R4) and (R5) as years went by and the computational resources improved.

The very first TDQMWP study of reaction (R3) was modest in its approach and scope. ${ }^{207}$ It investigated the dynamics of the exchange reaction in collinear geometry on a limited grid using a spline-fitted $a b$ initio PES and obtained average reaction probability values for the vibrational states $v=0,1,2$. Subsequently, TDQM studies ${ }^{208}$ were carried out on the MTJS surface $^{209,210}$ and it was shown that they could yield average reaction probability values in agreement with the averaged TIQM results on the same surface.

This study ${ }^{208}$ used the FFT method for evaluating the second derivative of the wave function with respect to the spatial coordinates and the second order finite difference method for evaluating the temporal derivatives. A special feature of this investigation was the use of different grid sizes $(64 \times 64,128 \times$ $64,128 \times 128$, and $256 \times 256)$ to ensure convergence of the results and minimization of errors due to reflection of the wave function from the edges of the grid. More refined studies were carried out on a $256 \times 128$ grid using the FFT route for the spatial derivatives and Chebyshev polynomial for the time propagation. ${ }^{211}$ Using the wave function splitting algorithm of Heather and Metiu ${ }^{117}$ and an absorbing boundary in the exit channel, ${ }^{99}$ energy resolved reaction probabilities were obtained for different vibrational states. In addition, the study mapped the quantal flux patterns during the course of the collision event. Subsequent studies by the same authors ${ }^{212}$ using a $192 \times$ 128 spatial grid and a Lanczos procedure ${ }^{213,214}$ for time evolution of the wave function coupled with time-energy mapping of the flux demonstrated the power of the TDQMWP method in reproducing the highly energy resolved reaction probability results for different vibrational states of $\mathrm{H}_{2}{ }^{+}$reported by Sakimoto and Onda $^{215}$ using the TIQM approach. Many of the oscillations in the plots of $P^{\mathrm{R}}$ versus $E_{\text {trans }}$ for the exchange reaction were identified earlier as Feshbach resonances arising from quasibound states supported by vibrationally adiabatic potentials. ${ }^{216}$ Quasiclassical trajectory calculations for the collinear $\left(\mathrm{He}, \mathrm{H}_{2}{ }^{+}\right)$reaction revealed bands of reactive and nonreactive trajectories and a number of chaotic trajectories with fractal characteristics. ${ }^{217}$

Time evolution of a chosen quantum mechanical wave packet on a $256 \times 256$ grid in mass-scaled Jacobi coordinates for the collinear geometry accompanied by computation of the autocorrelation function helped identify a number of transition state resonances. ${ }^{218} \mathrm{An}$ analysis of the eigenvalue spectrum for the quasibound states of collinear $\mathrm{HeH}_{2}{ }^{+}$revealed the signature of the underlying quantum chaos. ${ }^{219,220}$ Similar studies for collinear $\left(\mathrm{He}, \mathrm{HD}^{+}\right)$and $\left(\mathrm{He}, \mathrm{DH}^{+}\right)$revealed dramatically different behaviors. While the reaction probability for $\mathrm{HeH}^{+}$formation plotted as a function of energy ( $\left.E_{\text {trans }}\right)$ showed a ladder like structure, with each step corresponding to the opening up of a new product vibrational channel, $P^{\mathrm{R}}\left(E_{\text {trans }}\right)$ for $\mathrm{HeD}^{+}$formation showed a highly oscillatory structure. The transition state spectra for $\mathrm{HeHD}^{+}$and $\mathrm{HeDH}^{+}$revealed the characteristics of different dynamical behaviors in the two systems. ${ }^{219,221}$

Energy resolved reaction probabilities $P_{v, j}^{\mathrm{R}}\left(E_{\text {trans }}\right)$ for different $(v, j)$ states of $\mathrm{H}_{2}{ }^{+}$in collision with $\mathrm{He}$ in three dimensions were computed by solving the TDSE in mass scaled reactant channel Jacobi coordinates $(R, r, \gamma)$ for total angular momentum $J=0$ on a $128 \times 64 \times 32$ grid by integrating the flux across a dividing line. ${ }^{222}$ The $P_{v, j}^{R}\left(E_{\text {trans }}\right)$ results showed a number of oscillations indicative of the underlying reactive scattering resonances and also the vibrational enhancement that was observed in experiments.

While the FFT method was used for evaluating the second order spatial derivatives of the wave function with respect to $R$ and $r$, the DVR-FBR route was used for the angular part. The time evolution of the wave function was followed using the second order split-operator method. Similar studies were carried out for $\mathrm{He}, \mathrm{HD}^{+}$collisions in three dimensions ${ }^{223}$ using an L-shaped grid in $(R, r)$. In the interaction region, a $160 \times 128$ grid was used, while in the reactant channel an $80 \times 64$ grid was used. The angular grid consisted of 60 Gauss-Legendre quadrature points.

The computed $P_{v_{j}}^{\mathrm{R}}\left(E_{\text {trans }}\right)$ values showed fewer oscillations for $\mathrm{HeD}^{+}$formation than for $\mathrm{HeH}^{+}$. The calculations also pointed out the need to compute $P_{v, j}^{\mathrm{R}}\left(E_{\text {trans }}\right)$ values for nonzero $J$ and for $J$-averaging for the branching ratio between the $\mathrm{HeD}^{+}$ and $\mathrm{HeH}^{+}$channels to compare with experimental results.

An investigation of the dynamical resonances in $3 \mathrm{D} \mathrm{HeH}_{2}{ }^{+}$ and $\mathrm{HeHD}^{+}$systems for $J=0$ using the TDQMWP approach by Maiti et al. ${ }^{186}$ revealed one bound state for $\mathrm{HeH}_{2}{ }^{+}$and two pairs of bound states for $\mathrm{HeHD}^{+}$and a large number of quasibound states for both systems. While the quasibound states at low energies could be interpreted in terms of local modes, some of the higher energy state eigenfunctions were reminiscent of hyperspherical modes. Many higher energy states could not be assigned quantum numbers, indicating underlying unstable periodic orbits.

An elaborate TDQMWP study, within the CS approximation, by Maiti et al. ${ }^{224}$ yielded $P_{v, j}^{\mathrm{R}}\left(E_{\text {trans }}\right)$ values for $v=0-3, j=0$, and $J=0-35(45)$, and thence cross section $\left(\sigma_{v 0}^{\mathrm{R}}\left(E_{\text {trans }}\right)\right)$ values for the exchange reaction. Although the computed $\sigma_{v 0}^{\mathrm{R}}\left(E_{\text {trans }}\right)$ values showed oscillations, particularly for the higher $v$ states, they were in reasonable agreement with the experimental results. ${ }^{225,226}$

While most of the dynamical studies of $\mathrm{HeH}_{2}{ }^{+}$collisions were carried out on the MTJS PES till then, Panda and Sathyamurthy ${ }^{227}$ reported the results of TDQMWP studies on 
the newly reported PES by Palmieri et $a l^{228}$ for $v=0-3$ and $j=0$ for a range of $J$ values, within the CS approximation. They also showed a number of oscillations in the $P_{\nu 0}^{\mathrm{R}}\left(E_{\text {trans }}\right)$ curves, indicating the underlying dynamical resonances. There was noticeable dampening in the oscillations in the $P_{\nu 0}^{\mathrm{R}}\left(E_{\text {trans }}\right)$ curves with an increase in $J$. They also showed that the oscillations persisted in the $\sigma^{\mathrm{R}}\left(E_{\text {trans }}\right)$ curves, particularly for $v=3$, suggesting that they might be amenable to experimental observation.

Tiwari et al. ${ }^{229}$ revisited the problem of $\left(\mathrm{He}, \mathrm{HD}^{+}\right)$collisions and computed the reaction probabilities and integral reaction cross sections for $\mathrm{HeH}^{+}$and $\mathrm{HeD}^{+}$formation and their branching ratio for different vibrational states of $\mathrm{HD}^{+}$with $j=0$, averaged over $J$, within the CS approximation using the TDQMWP approach on the MTJS as well as the Palmieri et al. PES. Although $\mathrm{HeD}^{+}$formation was generally preferred over $\mathrm{HeH}^{+}$for different $v$ states of $\mathrm{HD}^{+}$over a range of $E_{\text {trans }}$ on both the PESs, there were noticeable (quantitative) differences between the dynamical outcomes on the two different surfaces. Unfortunately, the available experimental results are not adequate enough to decide in favour of the results on one surface or the other. The effect of reagent rotation on the branching ratio between $\mathrm{HeH}^{+}$and $\mathrm{HeD}^{+}$was investigated ${ }^{230}$ on the MTJS PES using the TDQMWP approach within the CS approximation by including nonzero $J$ values and the helicity quantum number $(K)$. The $P_{v, j}^{\mathrm{R}}\left(E_{\text {trans }}\right)$ values were highly oscillatory for both the channels. Yet, it was clear that $\mathrm{HeH}^{+}$formation was preferred over $\mathrm{HeD}^{+}$for large $J$ and $\mathrm{HeD}^{+}$was the preferred channel for small $J$ for all rotational states $(j=0,1,2,3)$. It is important to point out that the $P_{v, j}^{\mathrm{R}}\left(E_{\text {trans }}\right)$ values and hence partial cross section values depended strongly on $K$, thus necessitating $K$-averaging, in addition to $J$-averaging, while arriving at the reaction cross section values and hence the branching ratio for different $(v, j)$ states. In a more elaborate calculation ${ }^{135}$ including Coriolis coupling on the same PES, it was found that the results did not change with the inclusion of Coriolis coupling for the $\mathrm{HeD}^{+}$channel, but they depended heavily on $K$ for the $\mathrm{HeH}^{+}$channel. Kolakkandy ${ }^{9}$ has extended the calculations on a refined version of the Palmieri et al. PES published by Ramachandran et al. ${ }^{231}$ Although the final results are not much different between the MTJS PES and that of Ramachandran et al., ${ }^{231}$ the calculations reiterated the importance of Coriolis coupling.

Kolakkandy et al. ${ }^{116}$ carried out TDQMWP calculations within the CS approximation on the MTJS PES over an extended energy range (up to $4.7 \mathrm{eV}$ ) to investigate the competition between the exchange reaction (R3) and collision induced dissociation (R5) in ( $\left.\mathrm{He}, \mathrm{H}_{2}^{+}\right)$collisions for $v=0,1,2$ and $j=$ $0-3$. Although the computed results were in reasonable agreement with the available experimental results, ${ }^{225,226,232,233}$ they were strongly dependent on $K$. More calculations including Coriolis coupling on the Ramachandran et al. PES are clearly needed.

The number of triatomic and larger systems studied using the TDQMWP method has increased over the years as is evident from the reviews by Althorpe and Clary ${ }^{16}$ and Zhang and Guo. ${ }^{17}$
We have performed a limited survey of the systems investigated till 2004. ${ }^{198}$ The power and utility of the method is evident from the variety of systems investigated thus far: $\left.\left(\mathrm{H}, \mathrm{H}_{2}\right)\right)^{5,6,32,100,101,104,114,118,119,121,184} \quad(\mathrm{H}, \mathrm{HD}),{ }^{130,234}$ $\left(\mathrm{H}, \mathrm{D}_{2}\right),{ }^{120,123,134,235}\left(\mathrm{~F}, \mathrm{H}_{2}\left(\mathrm{HD}, \mathrm{D}_{2}\right)\right){ }^{134,236}\left(\mathrm{O}, \mathrm{H}_{2}\left(\mathrm{HD}, \mathrm{D}_{2}\right)\right){ }^{83,237}$ $\left(\mathrm{C}, \mathrm{H}_{2}\right),{ }^{238}\left(\mathrm{H}, \mathrm{O}_{2}\right),{ }^{125,132,139,239}\left(\mathrm{~N}, \mathrm{O}_{2}\right),{ }^{240}(\mathrm{O}, \mathrm{HCl}),{ }^{241}$ $(\mathrm{F}, \mathrm{HCl}),{ }^{242}(\mathrm{~N}, \mathrm{OH}),{ }^{243}(\mathrm{C}, \mathrm{OH}),^{244}(\mathrm{~S}, \mathrm{OH}),{ }^{245}\left(\mathrm{~N}, \mathrm{~N}_{2}\right){ }^{246}$ $\left(\mathrm{O}, \mathrm{O}_{2}\right),{ }^{247}(\mathrm{Li}, \mathrm{FH}),{ }^{120,248}\left(\mathrm{Li}, \mathrm{H}_{2}\right),{ }^{249}(\mathrm{H}, \quad \mathrm{LiH}),{ }^{250}$ $\left(\mathrm{H}, \quad \mathrm{LiH}^{+}\right),{ }^{251}\left(\mathrm{~N}, \quad \mathrm{H}_{2}\right),{ }^{252} \quad\left(\mathrm{H}^{-}, \mathrm{H}_{2}\right),{ }^{134,197,198,200-202,206,253}$ $\left(\mathrm{He}, \mathrm{H}_{2}^{+}\right),{ }^{8,105,116,131,135,207,208,211,212,222-224,227,229,230}\left(\mathrm{He}, \mathrm{HeH}^{+}\right),{ }^{254}$ $\left(\mathrm{He}, \mathrm{NeH}^{+}\right),{ }^{255}\left(\mathrm{Ne}, \mathrm{HeH}^{+}\right),{ }^{256}\left(\mathrm{Ne}, \mathrm{NeH}^{+}\right){ }^{257}(\mathrm{H}, \mathrm{HBr}){ }^{258}$ $(\mathrm{Br}, \mathrm{HD}),{ }^{259}\left(\mathrm{H}_{2}\left(\mathrm{HD}, \mathrm{D}_{2}\right), \mathrm{OH}\right){ }^{260}\left(\mathrm{H}, \mathrm{H}_{2} \mathrm{O}\right){ }^{261}(\mathrm{OH}, \mathrm{CO}){ }^{262}$ $\left(\mathrm{H}_{2}, \mathrm{CN}\right){ }^{263}(\mathrm{H}, \mathrm{HCN}(\mathrm{DCN})){ }^{264}\left(\mathrm{H}, \mathrm{CH}_{4}\right){ }^{265}\left(\mathrm{O}, \mathrm{CH}_{4}\right){ }^{266}$ $\left(\mathrm{Cl}, \mathrm{CH}_{4}\right),{ }^{267,268}$ and $\left(\mathrm{F}, \mathrm{CH}_{4}\right) .{ }^{269}$

The power of the TDQMWP method can be gauged further by a recent study of the reaction

$$
\mathrm{Cl}+\mathrm{HCH}_{3} \rightarrow \mathrm{ClH}+\mathrm{CH}_{3}
$$

in eight dimensions by Chen et al. ${ }^{268}$ that revealed oscillations when the reaction probability was plotted as a function of $E_{\text {trans }}$ for $J=0$ and could account for the experimentally observed backward scattering of the product $\left(\mathrm{CH}_{3}\right)$ species.

\subsection{Nonadiabatic interactions}

Unlike a spectroscopic study of bound molecular systems and reactive scattering on a single adiabatic PES, the study of scattering dynamics on coupled electronic states is much more complex and tedious. Much of the complexity arises due to the difficulty in representing the large amplitude molecular motion and asymptotic behaviour in a resonably acceptable diabatic electronic framework. It is only recently that some attempts have been made to investigate the effect of nonadiabatic coupling on photodissociation ${ }^{26,270-272}$ and bi-molecular reactive scattering ${ }^{273-287}$ dynamics. In the following we discuss the developments in the nonadiabatic dynamics study of (R1) in the recent past employing TDQMWP methods.

The electronic ground state of $\mathrm{H}_{3}$ in its equilateral triangle geometry is orbitally degenerate and it belongs to the $1^{2} \mathrm{E}^{\prime}$ electronic term of the $D_{3 \mathrm{~h}}$ symmetry point group. This electronic degeneracy is lifted on distortion of $\mathrm{H}_{3}$ along its degenerate bending and asymmetric stretching vibrational modes and the two split components form CIs in the $D_{3 \mathrm{~h}}$ symmetry configurations. This is the simplest and a classic example of an $E \otimes e$-JahnTeller system. ${ }^{288}$ The lower adiabatic component $\left(\mathrm{V}_{-}\right)$of the split $1^{2} \mathrm{E}^{\prime}$ surface is highly repulsive and the reaction dynamics occurs on it predominantly via a collinear reaction path. The upper adiabatic component $\left(\mathrm{V}_{+}\right)$, on the other hand, is bound in nature in the absence of its coupling to the lower surface. The seam of the CIs of the two component PESs occurs in the $D_{3 \mathrm{~h}}$ symmetry configuration of $\mathrm{H}_{3}$.

The repulsive lower adiabatic PES $\left(\mathrm{V}_{-}\right)$of $\mathrm{H}_{3}$ has been calculated by different groups ${ }^{289-292}$ with improved accuracy to study the dynamics of (R1). Among them, the double many body expansion (DMBE) PES of Varandas and coworkers ${ }^{290}$ represented both the adiabatic sheets of the $1^{2} E^{\prime}$ state of $\mathrm{H}_{3}$ for the first time. The DMBE PES is based on an analytic 
continuation technique that is found to be sufficiently accurate near the seam of the intersection of the two PESs. Recently, Yarkony carried out extensive $a b$ initio calculations of the adiabatic energies $\mathrm{V}_{-}$and $\mathrm{V}_{+}$and their nonadiabatic coupling terms. These $a b$ initio points were utilized by Abrol and Kuppermann $^{293,294}$ to develop new coupled diabatic PESs of the electronic ground state of $\mathrm{H}_{3}$. We have utilized the adiabatic energies of the DMBE PES along with the diabatization scheme of Thiel and Köppel ${ }^{173}$ to carry out the nuclear dynamics. In this scheme ${ }^{173}$ the diabatic potential energy matrix is given by

$$
\begin{aligned}
\left(\begin{array}{cc}
U_{11} & U_{12} \\
U_{21} & U_{22}
\end{array}\right) & =\mathbf{S}\left(\begin{array}{cc}
V_{-} & 0 \\
0 & V_{+}
\end{array}\right) \mathbf{S}^{\dagger} \\
& =\frac{V_{+}+V_{-}}{2} \mathbf{1}+\frac{V_{+}-V_{-}}{2}\left(\begin{array}{cc}
-\cos \chi & \sin \chi \\
\sin \chi & \cos \chi
\end{array}\right),
\end{aligned}
$$

where 1 is a $2 \times 2$ unit matrix and $\chi=2 \theta$ represents the polar angle in the two-dimensional Cartesian space of the degenerate vibration, called the pseudo-rotation angle. The topography of the adiabatic sheets of the JT-split electronic ground state of $\mathrm{H}_{3}$ is illustrated in Fig. 3.

Early work on the electronic nonadiabatic effects in the nuclear dynamics of $\mathrm{H}_{3}$ considered the inclusion of the GP in the single surface calculations. The transition state resonances of the lower adiabatic sheet $\left(\mathrm{V}_{-}\right)^{109,129}$ and the bound states of the upper adiabatic sheet $\left(\mathrm{V}_{+}\right)^{129}$ were studied using the time autocorrelation function approach. While no noticeable effect of the GP was found on the transition state resonances, the bound vibrational levels of the upper adiabatic sheet were found to undergo a considerable energy shift when the GP was included. This energy shift was first reported by Kuppermann and coworkers ${ }^{295}$ in a TIQM study. In addition to the energy shift, these authors also reported a change in the symmetry properties of the vibrational eigenstates of $\mathrm{V}_{+}$upon

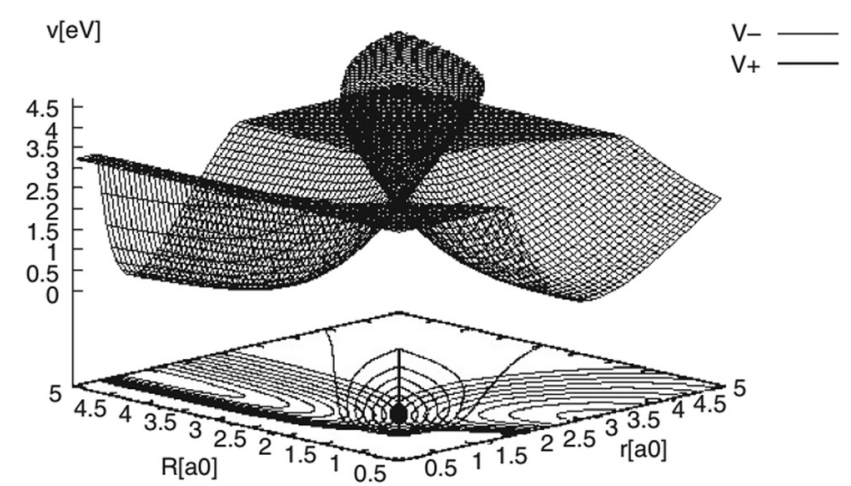

Fig. 3 The DMBE PES of the $1^{2} \mathrm{E}^{\prime}$ electronic states of $\mathrm{H}_{3}$ plotted in the Jacobi $(R, r)$ plane for $\gamma=\frac{\pi}{2}$. The contour line diagram is included at the base of the three-dimensional perspective plot. The lower $\left(V_{-}\right)$and upper $\left(V_{+}\right)$adiabatic states are shown by the thin and thick solid lines, respectively. The seam of the Cls of the two PESs occurring for $R=\sqrt{3 r} / 2$ is the straight line in the contour plot. The point on it indicates the energetic minimum of the seam occurring at $\sim 2.74 \mathrm{eV}$. inclusion of the GP. The effect of nonadiabatic coupling on the transition state resonances in the $\mathrm{H}+\mathrm{H}_{2}$ and $\mathrm{H}+\mathrm{D}_{2}$ reactions was also studied by Varandas and coworkers ${ }^{296,297}$ with a coupled diabatic model in a TDQMWP framework and it was found that the GP had practically no effect on the measurables.

Mahapatra and Köppel ${ }^{298}$ investigated the effect of nonadiabatic coupling on the transition state resonances of $\mathrm{V}_{-}$and bound vibrational levels of $\mathrm{V}_{+}$by devising a two-state diabatic model employing the adiabatic DMBE PESs. The calculations were carried out using the TDQMWP approach and the vibronic eigenvalue spectra and eigenfunctions were determined by the spectral quantization method. A detailed analysis revealed that resonances were not prominent in the neighborhood of the CIs and only a minor impact of nonadiabatic coupling on them showed up for energies below the minimum of the seam of the CIs occurring at $\sim 2.74 \mathrm{eV}$. This impact was attributed to the effect of the GP, which can be significant at energies below the intersection minimum. ${ }^{177}$ At an energy above this minimum some noticeable blurring of the eigenvalue spectrum was observed due to nonadiabatic coupling.

In contrast to the above, nonadiabatic coupling was found to have an extremely strong influence on the vibronic structure of $\mathrm{V}_{+}{ }^{298}$ The discrete bound vibrational level spectrum of $\mathrm{V}_{+}$in the absence of nonadiabatic coupling changes to a broad and structureless envelope with embedded resonances when the coupling is turned on. The bound states of $\mathrm{V}_{+}$were classified into $\mathrm{A}_{1}$ and $\mathrm{E}$ vibronic symmetry based on the nodal pattern of the corresponding eigenfunctions. ${ }^{298}$ While the eigenfunctions of $\mathrm{A}_{1}$ symmetry exhibit heavy build-up of probability density along the $D_{3 \mathrm{~h}}$ line (the intersection seam at $R=\sqrt{3 r} / 2$ in Fig. 3), the latter falls in the nodal plane of the eigenfunctions of E symmetry. The $\mathrm{A}_{1}$ symmetry levels are therefore immediately affected by the strong nonadiabatic coupling as compared to the levels of E symmetry in their decay dynamics in the coupled electronic manifold. The relaxation times of both types of vibronic levels are quantitatively estimated from the shorttime decay of the adiabatic electronic population as well as $|C(t)|$. It was found that the $\mathrm{A}_{1}$ levels decayed within $\sim 3$ fs and the E levels within $\sim 6$ fs, illustrating the mode specificity in the decay mechanism. ${ }^{298}$ Such an extremely fast decay time scale of molecular eigenstates was not known at that time and was in accordance with the phenomenological relaxation time of $\sim 2$ fs used by Bruckmeier et al. ${ }^{299}$ to simulate the experimental Rydberg emission spectrum of $\mathrm{D}_{3}$. Furthermore, in addition to the very fast mode specific decay of the vibronic levels of $\mathrm{V}_{+}$, the location of each peak appearing as a resonance in the broad spectral envelope shifted to a higher energy, supporting the earlier results that included the GP. ${ }^{295,296}$

To this end we would like to point out that the above work laid the foundation for a theoretical demonstration and interpretation of the recorded Rydberg emission spectrum of $\mathrm{H}_{3}$ and its deuterated isotopomers. ${ }^{300}$ The experimentally observed bimodal emission profile for the system was shown to arise from the lower and upper adiabatic sheets of the $1^{2} \mathrm{E}^{\prime}$ electronic ground state. Extremely strong nonadiabatic coupling and ultrafast internal conversion make the recorded spectrum 
completely structureless. ${ }^{300}$ It is clear that the effect of nonadiabatic coupling is strong on the vibronic structure of $\mathrm{V}_{+}$and the theoretical results corroborate the experimental observations extremely well.

It was discussed above that the nonadiabatic coupling operator $\boldsymbol{\Lambda}$ consists of three components viz., the $\mathrm{BH}$ correction term, the diverging derivative coupling term and the GP. Rajagopala Rao et al. ${ }^{127}$ examined the effect of each of these three terms in the recorded Rydberg emission spectrum of $\mathrm{D}_{3}{ }^{299}$ It turned out to be an appropriate (and perhaps the best) system to investigate as the emission experiment directly probes the dynamics of $\mathrm{D}_{3}$ at and in the immediate neighborhood of the CIs of the $1^{2} \mathrm{E}^{\prime}$ electronic ground state. The study was based on a TDQMWP approach in the modified hyperspherical coordinates of Johnson. ${ }^{47}$ The GP effect was incorporated into the formalism through the vector potential approach of Mead and Truhlar. ${ }^{150}$ The diagonal $\mathrm{BH}$ correction term assumes a simple form within a linear coupling approach and was shown to be given in terms of the magnitude $\left(\rho=\sqrt{q_{1}^{2}+q_{2}^{2}}\right.$, with $q_{1}$ and $q_{2}$ representing the mass-weighted cartesian components of the degenerate vibration) of the degenerate vibration of $D_{3}$ as ${ }^{184}$

$$
\Lambda^{0}=\frac{\hbar^{2}}{8 m_{\mathrm{D}} \rho^{2}}
$$

In eqn (56) $m_{\mathrm{D}}$ is the mass of the deuterium atom and $\rho$ represents the mass-weighted coordinate of the degenerate vibration in a polar representation. The complete nonadiabatic coupling was incorporated through the same diabatization ansatz of Thiel and Köppel ${ }^{173}$ as discussed above. It was shown that $^{127}$ the pseudorotation angle $\chi$ is equal to the hyperangle $\phi$ (cf. Appendix A in ref. 127).

The emission profile to the lower adiabatic sheet $\left(\mathrm{V}_{-}\right)$was broad and diffuse already when the states were not coupled. Inclusion of the coupling between $\mathrm{V}_{-}$and $\mathrm{V}_{+}$did not cause any significant change in the spectral profile. This is due to the repulsive nature of $\mathrm{V}_{-}$. Upon arrival in this state, the WP reaches various dissociation channels within $\sim 10$ fs. The discrete bound vibrational spectrum of uncoupled $\mathrm{V}_{+}$, on the other hand, was found to be strongly affected by the surface coupling (vide supra). Inclusion of both the GP and BH corrections caused a shift of each of its peaks to a higher energy without affecting the structure of the spectrum. It was found that both the GP and $\mathrm{BH}$ corrections caused nearly the same energy shift in the spectral peaks. The GP correction introduces a node in the nuclear wave function and the corresponding vibrational level shifts to a higher energy. The appearance of the node implies that the wave function does not encircle the CI. The BH term ( $c f$. , eqn (56)) is like a centrifugal term and it becomes singular at the CIs. It shifts the electronic potential to a higher energy and hence the underlying vibrational levels. Despite an overall energy shift, the detailed fine structure of the spectrum remained unaltered in this situation as compared to the uncoupled surface results. Upon inclusion of the NACTs, the dynamics on $\mathrm{V}_{+}$changed dramatically. The discrete vibrational level structure transformed to an extremely broad spectral envelope. While the inclusion of the GP and $\mathrm{BH}$ corrections shifted the envelope to a higher energy, the off-diagonal derivative coupling caused ultrafast internal conversion of the WP to the lower adiabatic sheet and a huge broadening of the spectral envelope. The minimum of the upper adiabatic sheet occurs at the minimum of the seam of the CIs. Therefore, upon arrival at this $\left(\mathrm{V}_{+}\right)$sheet, the WP is perturbed by the strong nonadiabatic coupling. The theoretical results discussed above were shown to be in accord with the experiment and therefore established that both the GP and $\mathrm{BH}$ corrections led to an energy correction. However, the most crucial effect of ultrafast relaxation of the molecular electronic state is caused by the off-diagonal elements of the nonadiabatic coupling operator. Complete surface coupling includes all the three effects on the dynamics in a coherent fashion. A summary of the results discussed above is given in Fig. 4. A movie containing the time evolution of the WP on the lower $\left(\mathrm{V}_{-}\right)$and upper $\left(\mathrm{V}_{+}\right)$adiabatic sheet of the electronic ground state of $\mathrm{D}_{3}$ under a strict $\mathrm{BO}$ approximation, including the $\mathrm{BH}$ and GP corrections and full surface coupling, can be downloaded from the URL http://chemistry.uohyd.ac.in/ sm/ SMGID/animation.html.

Early work on electronic nonadiabatic interactions in $\mathrm{H}+\mathrm{H}_{2}$ scattering considered the inclusion of GP change in the adiabatic electronic wave function in the nuclear dynamics on $V_{\text {- only. Mead }}{ }^{301}$ carried out the first study and proposed that inclusion of a phase factor of type $\mathrm{e}^{3 i \eta / 2}(\eta$, describes the coordinate of the path around the CI) is necessary for the single surface dynamics treatment owing to the existence of conical intersections on the $\mathrm{H}+\mathrm{H}_{2}$ PES and also to take care of the permutation symmetry of the identical nuclei. $\mathrm{He}^{301}$ pointed out that the GP changes the interference between the reactive and nonreactive scattering amplitudes of identical nuclei when compared to the non-GP case. More than a decade later, Kuppermann and coworkers ${ }^{302-306}$ carried out a series of more rigorous studies employing the multi-valued wave function basis approach of Mead and Truhlar ${ }^{150}$ and established that the GP significantly changed the differential reaction cross section (DCS). It was shown that the DCS of the $\mathrm{H}+\mathrm{D}_{2}(v=0)$ reaction at a collision energy of $1.26 \mathrm{eV}$ agreed well with the experiment when the GP effect was included. Later, in a number of combined experimental and theoretical studies ${ }^{307-312}$ it was shown that theoretical calculations without the GP could also reproduce the experimental results satisfactorily. This issue remained a subject of debate for some time. Kendrick ${ }^{313}$ performed TIQM calculations using the vector potential approach of Mead and Truhlar ${ }^{150}$ and showed that the effects of the GP were small and showed up in the state-to-state reaction probability above a total energy of $1.8 \mathrm{eV}$. The surprising result at that time was that the GP effects that appeared in the reaction probability results cancelled each other on summing over the partial wave contributions while arriving at the integral reaction cross section (ICS) values.

Althorpe and coworkers ${ }^{314}$ performed TDQMWP calculations using the vector potential approach of Mead and Truhlar and reached unequivocal agreement with the results of Kendrick. ${ }^{313}$ Althorpe and coworkers extended the calculations to larger $J$ values and found that small GP effects that showed 
(a)

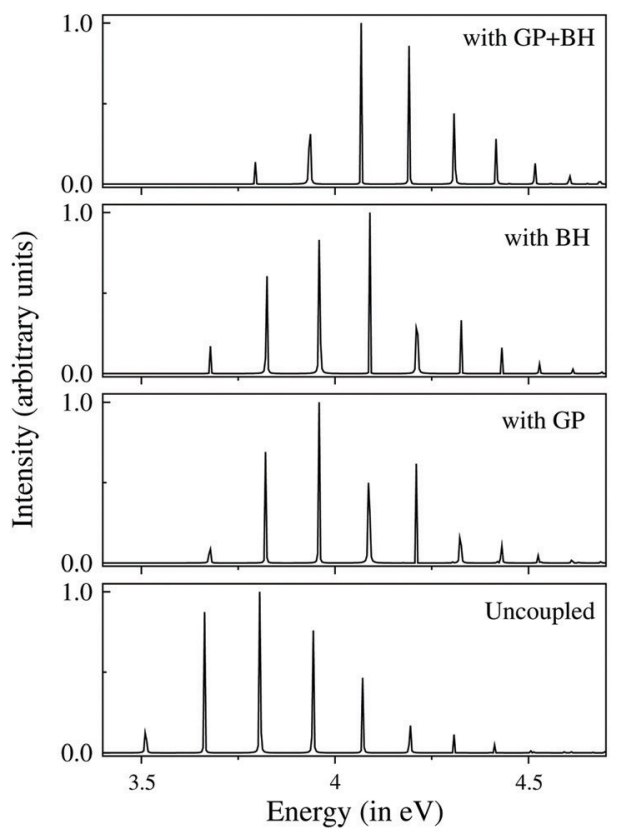

(b)

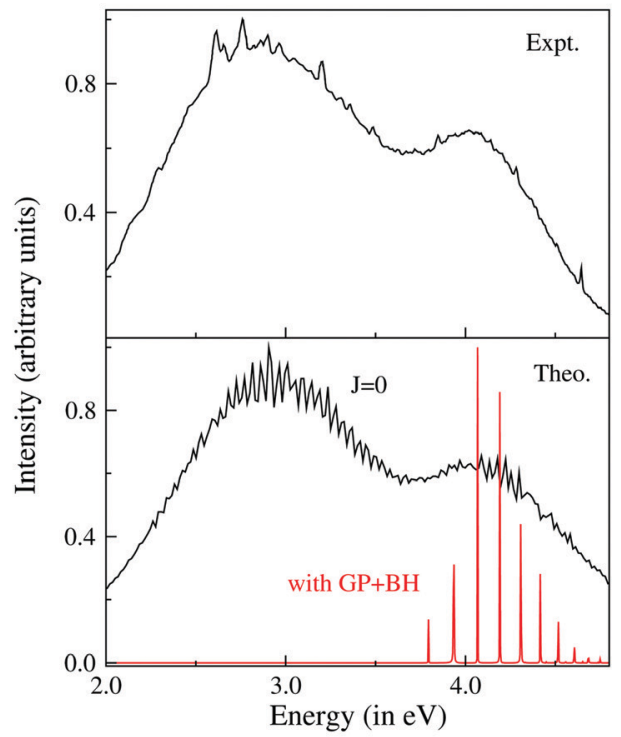

Fig. 4 Optical emission spectrum of $D_{3}$ from the $3 p^{2} E^{\prime}$ Rydberg electronic state to the upper adiabatic sheet of the JT split $1^{2} E^{\prime}$ electronic ground state. (a) The discrete vibrational energy level spectrum of uncoupled $\mathrm{V}_{+}$, with the GP, with the BH correction and with both the GP and BH corrections shown in the panels starting from the bottom, respectively. It is clear that both the GP and the BH correction cause an energy shift of each peak without affecting the spectral width. (b) Theoretical coupled-state results (the broad bimodal envelope in the bottom panel) compared to the experimental recording (top panel) are reproduced in ref. 127. The theoretical results of the top panel of (a) are included in the bottom panel of (b) to clearly indicate that both the GP and $\mathrm{BH}$ corrections are required to get the center-of-gravity of the spectrum at the right energy. The contribution of the nonadiabatic coupling to the spectral broadening of $V_{+}$is obvious from the plot.

up in the DCSs disappered in the ICS values. Subsequently, they $^{315}$ extended their study to higher (total) energies up to $4.5 \mathrm{eV}$. In addition to the GP, the authors also considered the inclusion of the $\mathrm{BH}$ correction and rigorous surface coupling following the theoretical model of Mahapatra et al. ${ }^{184}$ It was found that the $\mathrm{BH}$ term contributed to the dynamics at energies above $\sim 2.5 \mathrm{eV}$ as was found by Mahapatra et al. ${ }^{184}$ It was further demonstrated that the $\mathrm{BH}$ term, which is like a spike, protected the WP from the singularity of the vector potential and reduced the sideways scattering and the rotational temperature of the product $\mathrm{H}_{2} \cdot{ }^{315}$ The GP was shown to have strong effects on the DCS at energies above $\sim 3.5 \mathrm{eV}$, which however cancelled upon summing over all partial wave contributions to arrive at the ICS. These authors have also provided an explanation for such a cancellation by separating the contribution to the DCS from two different paths going over one and two transition states, respectively, and subsequent interference at high energies.

A summary of the above discussion is in order here. The lower adiabatic surface $\mathrm{V}_{-}$of $\mathrm{H}_{3}$ is highly repulsive and the WP readily accesses the dissociation channels both in spectroscopy and scattering studies. Reactive scattering is dominated by the collinear reaction path and resonances are not prominent in the neighborhood of the $D_{3 \mathrm{~h}}$ seam. The upper adiabatic sheet $\mathrm{V}_{+}$has its minimum at the minimum of the intersection seam at $\sim 2.74 \mathrm{eV}$. The dynamics of this state is, therefore, dramatically affected by the nonadiabatic interactions with $\mathrm{V}_{-}$.
While quite intense research activity on the effect of the GP on $\mathrm{H}+\mathrm{H}_{2}$ scattering dynamics still continues, ${ }^{316}$ Mahapatra et al. ${ }^{184}$ devised a theoretical model for the first time to include explicit surface coupling in a TDQMWP framework. In addition to computing reaction probabilities, the model was further extended to calculate ICSs and thermal rate constants. ${ }^{317,319,320}$ The stateselected $P^{\mathrm{R}}$ results for $(v=0, j=0)$ of $\mathrm{H}_{2}, J=0$, were examined over the energy range starting from the onset of the exchange reaction at $0.55 \mathrm{eV}$ to the three-body dissociation limit $(\sim 4.74 \mathrm{eV})$. It was found that the coupled electronic state results remained almost identical to the uncoupled state results. Only very small differences between the two were found beyond the energetic minimum of the seam of the CIs at $\sim 2.74 \mathrm{eV}$. This difference disappered when the $\mathrm{BH}$ correction was included in the uncoupled state calculations. In the adiabatic picture, the reactive flux of the WP flows to the product asymptote of the lower adiabatic sheet $\left(\mathrm{V}_{-}\right)$only. An analysis of the electron population revealed that less than $1 \%$ of the WP traverses the upper adiabatic sheet during the entire course of the dynamics. It was also found that the sum of the reaction probabilities obtained on the two diabatic product asymptotes was identical to that obtained in the adiabatic picture. This additionally reveals that the upper adiabatic sheet makes little contribution to the reaction dynamics in this case.

While the above results were obtained within the CS approximation, Jayachander Rao et al. included the CC terms of the Hamiltonian in a later study. ${ }^{317}$ The reaction probability for $\mathrm{H}_{2}$ $(v=0, j=0)$ was calculated up to a total energy of $3.0 \mathrm{eV}$. It was 
found that the location of the resonances shifted to higher energies as compared to the CS results. Such a shift was also reported by Padmanaban and Mahapatra ${ }^{318}$ in an earlier study. It was found that the difference between the uncoupled and coupled state results increased with an increase in $K$ for lower $J$ values. However, with an increase in $J$ this difference decreased, implying that CC did not promote nonadiabaticity in the $\mathrm{H}+\mathrm{H}_{2}$ dynamics. A similar observation of the CC effect on the uncoupled state dynamics with the GP was noted by Bouakline et al. ${ }^{315}$ Jayachander Rao et al. ${ }^{319}$ also studied the effect of reagent vibrational and rotational excitation on the nonadiabatic reactive dynamics of $\mathrm{H}+\mathrm{H}_{2}$. It was found that reagent vibrational excitation did not promote nonadiabaticity, whereas reagent rotational excitation did. The difference between the uncoupled and coupled surface results showed up in the reaction probability values and also in the ICSs, particularly for $\mathrm{H}_{2}(j=3)$. Interestingly, the difference was found to be quite substantial at energies below the minimum of the seam of the CIs for $j=3$. This was due to the GP effect (as stated above) even if the upper surface is inaccessible to the WP at these energies. No effect of surface coupling was found on the thermal rate constants.

Rajagopala Rao et $a .^{320}$ performed a detailed study of the dynamics of the $\mathrm{H}+\mathrm{D}_{2}, \mathrm{D}+\mathrm{H}_{2}, \mathrm{H}+\mathrm{HD}$ and $\mathrm{D}+\mathrm{HD}$ reactions. Reaction probabilities, ICSs and thermal rate constants were calculated with and without the NACTs for energies up to the three-body dissociation limit and also with vibrationally and rotationally excited reagents. No dramatic effect of nonadiabatic coupling was found on the dynamics of any of the four isotopic variants mentioned above. Both the ICSs and thermal rate constants were found to remain the same on the uncoupled and coupled surfaces and agreed well with the available experimental results. ${ }^{321}$ Lu et al. ${ }^{297}$ have reported ICSs of the $\mathrm{H}+\mathrm{D}_{2}$ reaction using the original diabatic DMBE PES of $\mathrm{H}_{3}{ }^{290}$ that compared very well with ours (up to a collision energy of $2 \mathrm{eV}$ ), implying that the diabatic DMBE PES of $\mathrm{H}_{3}$ is as accurate as the diabatization scheme employed by us.

In summary, it can be stated that the nonadiabatic coupling has similar effects on the reaction dynamics of $\mathrm{H}+\mathrm{H}_{2}$ and its isotopic variants. It was found by Althorpe and coworkers ${ }^{315}$ that the state-to-state DCSs calculated by including the GP and $\mathrm{BH}$ corrections were identical to the results obtained with the two state diabatic model over an extended energy range. It therefore emerges that the participation of the upper surface is minimal in the dynamics of the lower surface also for energies much above the minimum of the seam of the CIs and this still remains a mystery. More experimental and theoretical work are necessary to resolve this mystery. ${ }^{322}$

Some of the other systems that have been investigated using the TDQMWP method including non-adiabatic coupling are: $\left(\mathrm{H}^{+}, \mathrm{H}_{2}\right),{ }^{128,285,286}\left(\mathrm{~F}, \mathrm{H}_{2}\right){ }^{277,323}\left(\mathrm{Cl}, \mathrm{H}_{2}\right){ }^{32,279,324}\left(\mathrm{Br}, \mathrm{H}_{2}\right){ }^{325}$ $\left(\mathrm{O}, \mathrm{H}_{2}\right),{ }^{274,326}$ and more recently $\left(\mathrm{F}, \mathrm{CH}_{4}\right){ }^{287}$

\section{Large systems}

Despite the overwhelming success of the traditional TDQMWP method with the development of powerful integrators and improved algorithms, computational bottlenecks prevented its application to systems involving a large number $n$ of degrees of freedom (DOF). The number $N$ of nodes needed increases as $N^{n}$. Efforts have been made in the past to overcome this problem by devising both approximate and numerically exact methods applicable to large systems, with affordable computational cost. To name a few, the methods in the approximate category include the Gaussian wave packet (GWP) method of Heller, ${ }^{327}$ semiclassical approaches, ${ }^{328}$ the time-dependent self consistent field method ${ }^{329}$ and mixed classical and quantal approaches. ${ }^{330}$ In the numerically exact category path integral approaches, ${ }^{331}$ the multi-configuration time-dependent Hartree (MCTDH) method ${ }^{332}$ and self-consistent hybrid methods ${ }^{333}$ are noteworthy. Each of the above methods has its own limitations for it to be applicable to a given problem with a given Hamiltonian. Considerable efforts have been made in recent years to ensure the generality of some of these methods.

We mention selected methods from each of the two categories that use TDQMWP propagation. In the numerically exact category, the MCTDH method is the most promising one. It was originally formulated by Manthe, Meyer and Cederbaum and it emerged as a state-of-the-art technique to solve the TDSE numerically exactly on a grid for systems with large DOFs. It utilizes a DVR basis combined with the FFT algorithm and powerful integrators to propagate the WP. The crucial aspect of the method lies in the design of the initial WP, in contrast to the traditional grid method. The MCTDH method uses a product separable multi-configurational ansatz for the wave function in which each configuration is expressed as a Hartree product of time-dependent basis functions called single particle functions (SPFs). Several DOFs are combined to form the latter with a multi-set formulation and the WP is expanded in terms of them. This cuts down the computational cost heavily by effectively reducing the dimensionality of the problem. The MCTDH equations of motion are derived using the Dirac-Frenkel variational principle, ${ }^{334}$ ensuring an optimal description of the WP by the evolving SPFs. In that way the time-dependent basis moves with the WP, making the method efficient while keeping the basis size optimally small. For more technical details of the method and its applicability, the reader is referred to the URL: https://www.pci.uni-heidelberg.de/cms/ mctdh.html. We note that the complex reactive scattering problem $\mathrm{H}+\mathrm{CH}_{4}$ has been studied with the MCTDH method and the resulting reaction cross sections and rates were reported. ${ }^{335}$ The number of papers published (although the list is not exhaustive) utilizing the Heidelberg MCTDH program modules can be found in the given URL.

The MCTDH method mentioned above is further extended to a greater height by introducing variational flexibility in the initial trial wave function in a multilayer (ML) formulation called the ML-MCTDH method. This method was introduced by Wang and Thoss ${ }^{336}$ and the complex theoretical formulation was made implementable with the introduction of a recursive dynamically optimized layering scheme and layered correlation DVR by Manthe. ${ }^{337}$ In contrast to the original MCTDH method, which uses a static primitive basis to express the SPFs, the ML-MCTDH 
scheme uses a time-dependent expansion at different levels in the hierarchy. The ML-MCTDH scheme was shown to be quite efficient and successful to treat thousands of DOFs. The dynamics of the $\mathrm{H}+\mathrm{CH}_{4}$ reaction was also studied with the ML-MCTDH method by Welsch and Manthe. ${ }^{338}$ To deal with systems of identical particles and to account for exchange symmetry, multi-configuration time-dependent Hartree-Fock ${ }^{339}$ and multi-configuration time-dependent Hartree-Bose ${ }^{340}$ methods were formulated for Fermionic and Bosonic systems, respectively.

In the mixed classical and quantal approach of the approximate category, a large scale dynamical system is divided into two parts in which the most crucial DOFs are treated quantum mechanically in a numerically exact way and the rest of the DOFs are treated classically. Adhikari and co-workers ${ }^{341}$ have developed the algorithm called time-dependent DVR (TDDVR) in which the quantum part of the problem is treated in DVR basis and the WP propagation is carried out in a way similar to that in the MCTDH method. The classical part of the problem is treated by solving Newton's equations of motion. These authors have applied the method to a variety of problems that have been treated with the MCTDH approach and showed that the dynamical results from the two methods complemented each other.

At this point we mention two other promising methods developed for large systems, based on the idea underlying Gaussian wave packet dynamics. ${ }^{327,342}$ The first one, called the G-MCTDH method, developed by Burghardt and co-workers, ${ }^{343,344}$ utilizes the separation of the DOFs of the system in the spirit of the mixed classical and quantal approach. ${ }^{330}$ The large number of DOFs is divided into primary (strong coupling) and secondary (weak coupling) modes. The primary modes are treated with the numerically exact MCTDH equations of motion on a grid and the secondary modes are treated with an approximate propagation scheme, avoiding the grid representation. The latter, therefore, allows combining a large number of secondary modes into a package which is subsequently represented in terms of parameterized correlated multidimensional Gaussians. The Dirac-Frenkel variational principle ${ }^{334}$ is utilized to derive the equations of motion of the time dependent parameters. ${ }^{342}$ Here the method differs from the classical evolution of the Gaussian basis set ${ }^{327}$ and conforms to a semiclassical evolution. The correlation between the primary and the secondary modes is ensured in the propagation scheme employed and it also accounts for the quatum mechanical phase coherence. The method is developed both in the wave function and density matrix representations and extended to account for dissipative effects. It is potentially applicable to a wide variety of dynamical processes occurring in the gas phase as well as in the condensed phase and also to ones involving a thermal initial state.

The second promising method falls under the umbrella of $a b$ initio molecular dynamics. It is called the ab initio multiple spawning method (AIMS) and was developed by Martinez and collaborators. $^{345,346}$ In this method both the electronic and nuclear Schrödinger equations are solved simultaneously without separating the electronic structure and dynamics. In contrast to the methods discussed thus far, which require the multidimensional PES as a prerequisite, the PES is calculated on-thefly in the AIMS approach. It has a classical flavour in the sense that it connects the local quantum chemistry information to the global nature of the nuclear Schrödinger equation as the global PES is not available to the nuclei for their motion at any instance. The central feature of the method is to use an adaptive basis set, which is time-dependent and nonorthogonal. For a nonadiabatic problem, a new basis set is generated when the wave function approaches the neighbourhood of the coupling region of the PESs (spawning). The AIMS method attempts to solve the nuclear dynamics with a time-dependent basis evolved from the classical framework. ${ }^{327}$ The total wave function is expanded in terms of electronic wave functions dependent parametrically on the nuclear coordinates and the nuclear wave function dependent on the nuclear coordinates and time and timedependent coefficents. While the set of electronic wave functions is obtained from the quantum chemistry solution on-the-fly, the nuclear wave function is represented in terms of a superposition of multidimensional travelling frozen parameterized Gaussians with time-dependent coefficients. ${ }^{327}$ The time-dependent position and momentum parameters of the Gaussians are propagated with the aid of the classical Hamilton's equations of motion $^{327}$ and the nuclear phase is propagated semiclassicaly as an integral of the Lagrangian. The time-dependent coefficients of the total wave function are calculated variationally. The calculations are initiated by providing the basis set parameters (position, momentum and phase) drawn randomly from an appropriate Wigner distribution and in the beginning only one electronic state is populated. As the calculations progress new basis functions are spawned in a different electronic state while the parent basis passes through a region of nonadiabaticity. Complementary methods called variational multi-configurational Gaussian (vMCG) ${ }^{347}$ and coherent coupled states (CCS) are also developed within the framework of AIMS. The former is developed along the same lines as the G-MCTDH method of Burghardt and co-workers and is fully variational. The latter (the CCS method) developed by Shalashilin et al. ${ }^{348-350}$ falls in between the AIMS and vMCG methods. It has some additional quantum effects accounted for (as compared to AIMS) by averaging the trajectories over the Gaussians.

A summary and an outlook of the methods discussed for large systems are in order here. The MCTDH method is exactly quantum mechanical in nature globally and the rest of the methods are not. Approximations are made to alleviate the computational bottleneck. Heller's approach to the wave packet dynamics was a landmark in the field and it has led to significant advancements. A large number of complex systems have been treated by his method to bring in reasonable understanding of the dynamics on par with modern experiments. We believe that the developments noted above are still in their infancy and would evolve further with time.

\section{Molecular dynamics on solid surfaces}

The dynamics of molecules on solid surfaces is more complex than in the gas phase as one has to deal with not only the 
molecular (electronic and vib-rotational) degrees of freedom, but also the interaction with the surface and the characteristics of the solid surface. While some of the earlier TDQMWP studies $^{351}$ on $\mathrm{H}_{2} / \mathrm{Cu}$, for example, treated the solid surface as rigid and flat, subsequent studies introduced corrugation. Kroes and collaborators ${ }^{352}$ have investigated the dynamics of $\mathrm{H}_{2}$ dissociation on the $\mathrm{Cu}(111)$ surface using a chemically accurate potential. Gerrits et al. ${ }^{353}$ combined the power of the WP method with the utility of the neural network approach for potential determination for $\mathrm{CHD}_{3} / \mathrm{Cu}(111)$. Tiwari et al. ${ }^{354}$ have shown that the lattice motion influences the reactivity of the methane molecule on the nickel (111) surface. The same set of authors $^{355}$ reiterated their findings for dissociative chemisorption of methane on nickel and platinum surfaces using the reaction path Hamiltonian method. ${ }^{356}$ Guo and collaborators $^{357,358}$ examined the role of vibrational excitation in the dissociative chemisorption of water on copper and nickel surfaces using density functional theory based potentials and six dimensional TDQMWP studies. The application of the TDQMWP method to study the dissociation of water and methane molecules on $\mathrm{Cu} / \mathrm{Ni}$ surfaces has been reviewed recently by Tiwari and coworkers. ${ }^{359}$ The 9-dimensional study of methane dissociation on metal surfaces by Zhang and Jiang $^{360}$ is particularly worth mentioning.

\section{Software development}

The TIQM method had matured to a level that the $(\mathrm{A}+\mathrm{BC})$ reactive scattering code was made available as early as the year $2000 .^{199}$ Subsequently, public domain software under the name Dynasol ${ }^{361}$ was made available by J. Z. H. Zhang in 2004 for carrying out TDQMWP calculations. Software named DIFFREALWAVE ${ }^{137}$ of Hankel and Balint-Kurti was made available in 2008 to carry out RWP calculations.

\subsection{Machine learning and quantum dynamics}

The TDQMWP approach relies on (i) generation of an ab initio PES for a given system, (ii) fitting an analytic function to or numerical interpolation/extrapolation of or a combination of both of the generated points on the PES and (iii) solving the TDSE on a spatial grid for a length of time and extracting the dynamical information and the observables. When we go beyond the BO approximation, a manifold of PESs needs to be generated and nonadiabatic coupling included before the multi-state quantum dynamics is carried out. Recent developments of machine learning (ML) tools in the form of neural networks, Gaussian processes and kernel methods could enable the generation of the PES for moderate size $(3 \leq N \leq 6)$ systems, using a limited number of $a b$ initio points in $3 N-6$ dimensional space. Going beyond the BO approximation does not seem to be a serious bottleneck in this endeavour. The possibility of extending the ML tools to estimate observables like ( $l$-averaged) reaction cross sections and (ensemble averaged) reaction rate coefficients along with error estimates based on Bayesian optimization opens up new horizons. ${ }^{362}$ When these tools become more practical, the entire area of $a b$ initio quantum dynamics will undergo a sea change and more and more systems would be amenable to theoretical/computational investigations.

\section{Summary and conclusion}

We have outlined in this article the evolution of the TDQMWP methodology from its first use in predicting the rate coefficients for a model collinear $\left(\mathrm{H}, \mathrm{H}_{2}\right)$ exchange reaction to predicting the state-to-state differential and integral reaction cross sections for reaction (R1) in three dimensions on an accurate ab initio PES. That the predicted observables are in agreement with the available experimental results bears testimony to the reliability of the method. That the method has been extended to a number of atom-diatom exchange reactions and collision induced processes points out its practical utility. That the method is extendable to larger polyatomic systems either on its own or in conjunction with other methods demonstrates its versatility. That the method can be extended readily to include nonadiabatic coupling terms to predict vibronic interactions indicates its power. That the method is amenable to the use of machine learning tools in its implementation assures its future in the hands of theoretical spectroscopists and reaction dynamicists.

For some time, it appeared that the TDQMWP method had reached its limit and would not be applicable to systems involving more than three atoms or three dimensions because the number of grid points required along the approach coordinate in an exchange reaction or the retreat coordinate in photo-dissociation for obtaining reaction attributes with spectroscopic accuracy is large. One cannot afford to use the same number of grid points along all other degrees of freedom, in view of the increased demand on computer time and memory. The combination of the FFT route for evaluating the Laplacian of the wave function with Chebyshev polynomial expansion for the (time) evolution operator yielded results to machine accuracy. Although the FFT method scaled as $N \ln N$, where $N$ is the number of grid points, the DVR method came in as an attractive alternative, despite its scaling as $N^{2}$, the reason being that the latter method could be more efficient if an appropriate basis set is chosen for expanding the wave function. The second order split operator method for time evolution was robust enough and it could provide insight into the dynamics during the entire course of time evolution for several dynamical systems. With the development of higher order SO methods, the accuracy and stability of the algorithm ceased to be an issue. As we have shown in the main body of the text, the time evolution required of many dynamical studies of reactive scattering, photodissociation, gas-surface scattering, etc. is at most of the order of a few picoseconds and seldom of the order of nanoseconds. In addition to the grid size, the bottleneck in the application of the grid method that remained was the lack of availability of multidimensional PESs for larger (more than three or four atoms) systems. Once that was overcome by using methods such as permutationally invariant polynomials and artificial neural networks or a combination of the two, the wave packet method got 
a new life. Parallelization of computer codes definitely helped in dealing with systems that required calculations for a large number of $J$ states, even for triatomic systems.

In the case of photo-excitation processes also, three atom systems remained the norm for some time. When it came to gas-surface scattering, two or three spatial dimensions were the norm until a few years ago. Practitioners in the field have come up with clever ways to include lattice vibrations in molecule-solid surface interactions. Here again, the neural network methods are beginning to facilitate the extension of the method to surfaces involving more than a few atoms.

Alternative methods such as MCTDH and its variants and other methods such as TDDVR, AIMS, vMCG, CCS etc. have made the time-dependent approach practical for a variety of multi-dimensional systems.

Going beyond the BO approximation, dealing with dynamics that involves several electronic states and nonadiabatic coupling between them in more than one or two dimensions is a challenge. To start with, multi-sheet potential energy surfaces with their crossings and/or avoided crossings have to be determined in a readily usable form. In principle, one could take the diabatic route and use the existing TDQMWP methodology for solving multi-electronic state problems. In practice, it is a demanding task and it has been accomplished for a handful of systems (only). Mercifully, the computational time required for following the nuclear dynamics in such systems scales approximately linearly with an increase in the number of electronic states. Like in any scientific enterprise, as the challenges come up, solutions emerge too!

\section{Conflicts of interest}

There are no conflicts to declare.

\section{Acknowledgements}

We are grateful to Aditya Narayan Panda of IIT Guwahati for the graphic illustration of the evolution of the wave packet for $\left(\mathrm{He}, \mathrm{H}_{2}^{+}\right.$) dynamics in collinear and in 3D geometries. S. M. acknowledges the financial support from the Department of Science and Technology, New Delhi, through the grant no. EMR/2017/004592 and from the University Grants Commission (UGC-BSR mid career award grant No. F.19-231/2018(BSR)), New Delhi. Computational facilities availed in the CMSD, University of Hyderabad, are gratefully acknowledged. We thank Mr Jayakrushna Sahoo for help with the production of the manuscript.

\section{References}

1 E. Schrödinger, Ann. Phys., 1926, 4(79), 361-376.

2 E. Schrödinger, Ann. Phys., 1926, 4(79), 489-527.

3 E. Schrödinger, Ann. Phys., 1926, 4(81), 109-139.

4 P. A. M. Dirac, Proc. R. Soc. A, 1929, 123, 714-733.

5 J. Mazur and R. J. Rubin, J. Chem. Phys., 1959, 31, 1395-1412.
6 E. A. McCullough and R. E. Wyatt, J. Chem. Phys., 1969, 51, 1253-1254; E. A. McCullough and R. E. Wyatt, J. Chem. Phys., 1971, 54, 3578-3591; E. A. McCullough and R. E. Wyatt, J. Chem. Phys., 1971, 54, 3592-3600.

7 G. E. Kellerhals, N. Sathyamurthy and L. M. Raff, J. Chem. Phys., 1976, 64, 818-825.

8 A. N. Panda and N. Sathyamurthy, Proc. Indian Natl. Sci. Acad., Part A, 2004, 70, 635-648.

9 S. Kolakkandy, PhD thesis, Indian Institute of Technology Kanpur, 2012.

10 M. Levitt, Ernst Chain Lecture, Imperial College, London, 2014, https:/www.youtube.com/watch?v=Wy0OGgmjzPM.

11 L. R. Khundkar and A. H. Zewail, Annu. Rev. Phys. Chem., 1990, 41, 15-60.

12 A. H. Zewail and J. M. Thomas, 4D Electron Microscopy, Imperial College Press, London, 2010.

13 T. S. Rose, M. J. Roskar and A. H. Zewail, J. Chem. Phys., 1989, 91, 7415-7436.

14 F. T. Wall, L. A. Hiller Jr. and J. Mazur, J. Chem. Phys., 1958, 29, 255-263.

15 R. N. Porter and L. M. Raff, in Dynamics of Molecular Collisions Part B, ed. W. H. Miller, Plenum, New York, 1976, ch. 1.

16 S. C. Althorpe and D. C. Clary, Annu. Rev. Phys. Chem., 2003, 54, 493-529.

17 D. H. Zhang and H. Guo, Annu. Rev. Phys. Chem., 2016, 67, 135-58.

18 N. Sathyamurthy, in Reaction Dynamics: Recent Advances, ed. N. Sathyamurthy, Narosa, New Delhi, 1991, ch. 2.

19 A. H. Zewail, The Chemical Bond: Structure and Dynamics, Academic Press, San Diego, 1992.

20 Y. T. Lee, Angew. Chem., 1987, 26, 939-1058.

21 F. Fernández-Alonso and R. N. Zare, Annu. Rev. Phys. Chem., 2002, 53, 67-99.

22 M. Shapiro and P. Brumer, Principles of the Quantum Control of Molecular Processes, Wiley-VCH, 2003.

23 D. Neuhauser and H. Rabitz, Acc. Chem. Res., 1993, 26, 496-501.

24 D. J. Tannor, Introduction to Quantum Mechanics: A timedependent perspective, University Science Books, Sausalito, CA, 2007.

25 G. G. Balint-Kurti, S. Zou and A. Brown, Adv. Chem. Phys., 2008, 138, 43-94.

26 R. Schinke, Photodissociation Dynamics, Cambrige Univ. Press, Cambridge, 1993.

27 K. Ramasesha, S. R. Leone and D. M. Neumark, Annu. Rev. Phys. Chem., 2016, 67, 41-63.

28 V. Mohan and N. Sathyamurthy, Curr. Sci., 1986, 55, 115-119.

29 V. Mohan and N. Sathyamurthy, Comput. Phys. Rep., 1988, 7, 213-258.

30 N. Balakrishnan, C. Kalyanaraman and N. Sathyamurthy, Phys. Rep., 1997, 280, 79-144.

31 S. Mahapatra, N. Chakrabarti and N. Sathyamurthy, Int. Rev. Phys. Chem., 1999, 18, 235-262.

32 S. Mahapatra, Int. Rev. Phys. Chem., 2004, 23, 483-512. 
33 R. B. Gerber, R. Kosloff and M. Berman, Comput. Phys. Rep., 1986, 5, 59-114.

34 R. Kosloff, J. Phys. Chem., 1988, 92, 2087-2100.

35 Thematic issue on time-dependent methods for quantum dynamics, in Comput. Phys. Commun., ed. K. C. Kulander, 1991, vol. 63.

36 J. Z. H. Zhang, Theory and application of quantum molecular dynamics, World Scientific, Singapore, 1999.

37 G. G. Balint-Kurti, Adv. Chem. Phys., 2003, 128, 249-301;

G. G. Balint-Kurti, Int. Rev. Phys. Chem., 2008, 27, 507-539.

38 C. Leforestier, Chem. Phys., 1984, 87, 241-261.

39 S. Choi and J. Vaníček, J. Chem. Phys., 2019, 151, 234102.

40 E. B. Wilson, J. C. Decius and P. C. Cross, Molecular Vibrations, Dover, New York, NY, 1955.

41 S. Glasstone, K. J. Laidler and H. Eyring, The theory of rate processes, McGraw-Hill, New York, 1941, p. 101.

42 L. M. Delves, Nucl. Phys., 1959, 9, 391-399; L. M. Delves, Nucl. Phys., 1960, 20, 275-308.

43 P. M. Morse and H. Feshbach, Methods of Theoretical Physics, McGraw-Hill, New York, 1953, p. 1728-1734.

44 F. T. Smith, J. Math. Phys., 1962, 3, 735-748.

45 R. C. Whitten and F. T. Smith, J. Math. Phys., 1968, 9, 1103-1113.

46 A. Kuppermann, Chem. Phys. Lett., 1975, 32, 374-375.

47 B. R. Johnson, J. Chem. Phys., 1980, 73, 5051-5058.

48 T. Seideman and W. H. Miller, J. Chem. Phys., 1992, 96, 4412-4422; T. Seideman and W. H. Miller, J. Chem. Phys., 1992, 97, 2499-2514.

49 U. Manthe and W. H. Miller, J. Chem. Phys., 1993, 99, 3411-3419.

50 J. Z. H. Zhang and D. H. Zhang, J. Chem. Phys., 1994, 101, 1146-1156.

51 A. J. H. M. Meijer and E. M. Goldfield, J. Chem. Phys., 1998, 108, 5404-5413.

52 E. M. Goldfield and S. K. Gray, Comput. Phys. Commun., 1996, 98, 1-14.

53 R. T Pack, J. Chem. Phys., 1974, 60, 633-639.

54 P. McGuire and D. J. Kouri, J. Chem. Phys., 1974, 60, 2488-2499.

55 R. Dawes and S. A. Ndengué, Int. Rev. Phys. Chem., 2016, 35, 441-478.

56 N. Sathyamurthy, Comput. Phys. Rep., 1985, 3, 1-70.

57 J. N. Murrell, S. Carter, S. C. Farantos, P. Huxley and A. J. C. Varandas, Molecular Potential Energy Functions, Wiley, Chichester, 1984.

58 G. C. Schatz, Rev. Mod. Phys., 1989, 61, 669-688.

59 P. M. Morse, Phys. Rev., 1929, 34, 57-64.

60 F. London, Probleme der Modernen Physik (SommerfeldFestschrift), S. Hirzel, Leipzig, 1928, p. 104; F. London, Z. Elektrochem., 1929, 35, 552-555.

61 H. Eyring and M. Polanyi, Z. Phys. Chem. B, 1931, 12, 279-311; H. Eyring and M. Polanyi, Z. Phys. Chem., 2013, 227, 1221-1245.

62 J. C. Polanyi and J. L. Schreiber, in Physical Chemistry, an Advanced Treatise, Kinetics of Gas Reactions, ed. H. Eyring, W. Jost and D. Henderson, Academic Press, New York, 1974, ch. 6, vol. VIA.
63 P. J. Kuntz, Chem. Phys. Lett., 1972, 16, 581-583.

64 K. S. Sorbie and J. N. Murrell, Mol. Phys., 1975, 29, 1387-1407.

65 A. Aguado, C. Tablero and M. Paniagua, Comput. Phys. Commun., 1998, 108, 259-266.

66 C. M. R. Rocha and A. J. C. Varandas, Comput. Phys. Commun., 2020, 247, 106913.

67 B. J. Braams and J. M. Bowman, Int. Rev. Phys. Chem., 2009, 28, 577-606.

68 B. Jiang, J. Li and H. Guo, Int. Rev. Phys. Chem., 2016, 35, 479-506.

69 C. Qu, Q. Yu and J. M. Bowman, Annu. Rev. Phys. Chem., 2018, 69, 151-175.

70 L. M. Raff, R. Komanduri, M. Hagan and S. T. S. Bukkapatnam, Neural Networks in Chemical Reaction Dynamics, Oxford Univ. Press, Oxford, 2012.

71 M. Majumder, S. A. Ndengué and R. Dawes, Mol. Phys., 2015, 114, 1-18.

72 A. Kamath, R. A. Vargas-Hernández, R. V. Krems, T. Carrington and S. Manzhos, J. Chem. Phys., 2018, 148, 241702.

73 Y. Guan, S. Yang and D. H. Zhang, Mol. Phys., 2018, 116, 823-834.

74 N. Sathyamurthy and L. M. Raff, J. Chem. Phys., 1975, 63, 464-473.

75 M. Patrício, J. L. Santos, F. Patrício and A. J. C. Varandas, J. Math. Chem., 2013, 51, 1729-1746.

76 H. Nakamura, Nonadiabatic transition, World Scientific, Singapore, 2002.

77 Conical Intersections: Electronic Structure, Dynamics and Spectroscopy, ed. W. Domcke, D. R. Yarkony and H. Köppel, World Scientific, Singapore, 2004, vol. 15.

78 M. Baer, Beyond Born-Oppenheimer: Conical intersections and electronic nonadiabatic coupling terms, John Wiley \& Sons, Hoboken, New Jersey, USA, 2006.

79 T. Yonehara, K. Hanasaki and K. Takatsuka, Chem. Rev., 2012, 112, 499-542.

80 M. S. Schuurman and A. Stolow, Annu. Rev. Phys. Chem., 2018, 69, 427-450.

81 B. Mukherjee, K. Naskar, S. Mukherjee, S. Ghosh, T. Sahoo and S. Adhikari, Int. Rev. Phys. Chem., 2019, 38, 287-341.

82 A. J. C. Varandas, in ref. 77, pp. 205-270.

83 M. Hankel, G. G. Balint-Kurti and S. K. Gray, J. Phys. Chem. A, 2001, 105, 2330-2339; M. Hankel, G. G. Balint-Kurti and S. K. Gray, Int. J. Quantum Chem., 2003, 92, 205-211.

84 M. D. Feit, J. A. Fleck Jr. and A. Steiger, J. Comput. Phys., 1982, 47, 412-433.

85 H. Tal-Ezer and R. Kosloff, J. Chem. Phys., 1984, 81, 3967-3971.

86 Z. Sun, W. Yang and D. H. Zhang, Phys. Chem. Chem. Phys, 2012, 14, 1827-1845.

87 M. Braun, C. Meier and V. Engel, Comput. Phys. Commun., 1996, 93, 152-158.

88 P. Pfeifer and R. D. Levine, J. Chem. Phys., 1983, 79, 5512-5519.

89 U. Peskin and N. Moiseyev, J. Chem. Phys., 1993, 99, 4590-4596; U. Peskin, O. E. Alon and N. Moiseyev, 
J. Chem. Phys., 1994, 100, 7310-7318; U. Peskin, R. Kosloff and N. Moiseyev, J. Chem. Phys., 1994, 100, 8849-8855; N. Moiseyev, M. Chrysos and R. Lefebvre, J. Phys. B: At., Mol. Opt. Phys., 1995, 28, 2599-2609.

90 P. Raj, A. Gugalia and P. Balanarayan, J. Chem. Theory Comput., 2020, 16, 35-50.

91 M. D. Feit and J. A. Fleck Jr., J. Chem. Phys., 1982, 78, 301-308.

92 D. Kosloff and R. Kosloff, J. Comput. Phys., 1983, 52, 35-53. 93 J. Manz, private communication.

94 R. C. Mowrey and D. J. Kouri, J. Chem. Phys., 1986, 84, 6466-6473.

95 R. C. Mowrey, J. Chem. Phys., 1991, 94, 7098-7105.

96 D. H. Zhang and J. Z. H. Zhang, J. Chem. Phys., 1994, 101, 3671-3678; D. Neuhauser, M. Baer, R. S. Judson and D. J. Kouri, J. Chem. Phys., 1990, 93, 312-322; D. Neuhauser, Chem. Phys. Lett., 1992, 200, 173-178.

97 C. Leforestier and R. E. Wyatt, J. Chem. Phys., 1983, 78, 2334-2344.

98 R. Kosloff and D. Kosloff, J. Comput. Phys., 1986, 63, 363-376.

99 D. Neuhauser and M. Baer, J. Chem. Phys., 1989, 90, 4351-4355.

100 D. Neuhauser, M. Baer, R. S. Judson and D. J. Kouri, J. Chem. Phys., 1989, 90, 5882-5884.

101 D. Neuhauser, M. Baer and D. J. Kouri, J. Chem. Phys., 1990, 93, 2499-2505.

102 M. S. Child, Mol. Phys., 1991, 72, 89-93.

103 Á. Vibók and G. G. Balint-Kurti, J. Phys. Chem., 1992, 96, 8712-8719.

104 M. Monnerville, P. Halvick and J. C. Rayez, Chem. Phys., 1992, 159, 227-234.

105 S. Mahapatra and N. Sathyamurthy, J. Chem. Soc., Faraday Trans., 1997, 93, 773-779.

106 C. Leforestier, R. Bisseling, C. Cerjan, M. D. Feit, R. Friesner, A. Gulberg, A. Hammerich, G. Jolicard, W. Karrlein, H.-D. Meyer, N. Lipkin, O. Roncero and R. Kosloff, J. Comput. Phys., 1991, 94, 59-80.

107 T. N. Truong, J. J. Tanner, P. Bala, J. A. McCammon, D. J. Kouri, B. Lesyng and D. K. Hofmann, J. Chem. Phys., 1992, 96, 2077-2084.

108 J. V. Lill, G. A. Parker and J. C. Light, Chem. Phys. Lett., 1982, 89, 483-489; J. C. Light, I. P. Hamilton and J. V. Lill, J. Chem. Phys. , 1985, 82, 1400-1409; Z. Bačić and J. C. Light, Annu. Rev. Phys. Chem., 1989, 40, 469-498.

109 R. T. Skodje, R. Sadeghi, H. Köppel and J. L. Krause, J. Chem. Phys., 1994, 101, 1725-1729.

110 U. Manthe, PhD thesis, Universität Heidelberg, 1991; V. Engel, Chem. Phys. Lett., 1992, 189, 76-78; U. Manthe, H.-D. Meyer and L. S. Cederbaum, J. Chem. Phys., 1992, 97, 9062-9071.

111 W. H. Press, W. P. Flannery, S. A. Teukolksy and W. T. Vetterling, Numerical Recipes, Cambridge Univ. Press, 1986.

112 A. B. Myers, Acc. Chem. Res., 1996, 30, 519-527.

113 S. Mukamel, Principles of nonlinear optics, Oxford Univ. Press, Oxford, 1995.
114 S. C. Althorpe, J. Chem. Phys., 2001, 114, 1601-1616.

115 J. J. Sakurai, in Modern Quantum Mechanics, ed. S. F. Tuan, Addison-Wesley publishing company, 1994, p. 101.

116 S. Kolakkandy, K. Giri and N. Sathyamurthy, J. Chem. Phys., 2012, 136, 244312.

117 R. Heather and H. Metiu, J. Chem. Phys., 1987, 86, 5009-5017.

118 T. Peng and J. Z. H. Zhang, J. Chem. Phys., 1996, 105, 6072-6074; W. Zhu, T. Peng and J. Z. H. Zhang, J. Chem. Phys., 1997, 106, 1742-1748.

119 R. S. Judson, D. J. Kouri, D. Neuhauser and M. Baer, Phys. Rev. A: At., Mol., Opt. Phys., 1990, 42, 351-366; S. Y. Lin and H. Guo, Phys. Rev. A: At., Mol., Opt. Phys., 2006, 74, 022703; S. Gómez-Carrasco and O. Roncero, J. Chem. Phys., 2006, 125, 054102.

120 A. Zanchet, O. Roncero, T. González-Lezana, A. RodríguezLópez, A. Aguado, C. Sanz-Sanz and S. Gómez-Carrasco, J. Phys. Chem. A, 2009, 113, 14488-14501.

121 M. Hankel, S. C. Smith, R. J. Allan, S. K. Gray and G. G. Balint-Kurti, J. Chem. Phys., 2006, 125, 164303.

122 S. K. Gray and G. G. Balint-Kurti, J. Chem. Phys., 1998, 108, 950-962.

123 T.-S. Chu, K.-L. Han, M. Hankel and G. G. Balint-Kurti, J. Chem. Phys., 2007, 126, 214303.

124 M. Hankel, S. C. Smith and A. J. C. Varandas, Phys. Scr., 2011，84，028102; H. Yang, K.-L. Han, G. C. Schatz, S.-H. Lee, K. Liu, S. C. Smith and M. Hankel, Phys. Chem. Chem. Phys., 2009, 11, 11587-11595; P. G. Jambrina, F. J. Aoiz, N. Bulut, S. C. Smith, G. G. Balint-Kurti and M. Hankel, Phys. Chem. Chem. Phys., 2010, 12, 1102-1115; R. Lü, T.-S. Chu, Z.-S. Chang and W.-Q. Zhang, Bull. Chem. Soc. Jpn., 2014, 87, 670-676; Z. Shen, H. Ma, C. Zhang, M. Fu, Y. Wu, W. Bian and J. Cao, Nat. Commun., 2017, 8, 14094.

125 Z. Sun, X. Xu, S. Y. Lee and D. H. Zhang, J. Phys. Chem. A, 2009, 113, 4145-4154.

126 Z. Sun, H. Guo and D. H. Zhang, J. Chem. Phys., 2010, 132, 084112.

127 T. Rajagopala Rao and S. Mahapatra, J. Chem. Phys., 2011, 134, 204307.

128 S. Ghosh, S. Mukherjee, B. Mukherjee, S. Mandal, R. Sharma, P. Chaudhury and S. Adhikari, J. Chem. Phys., 2017, 147, 074105.

129 A. J. C. Varandas and H. G. Yu, J. Chem. Soc., Faraday Trans., 1997, 93, 819-824.

130 H. Zhao, X. Hu, D. Xie and Z. Sun, J. Chem. Phys., 2018, 149, 174103.

131 N. Balakrishnan and N. Sathyamurthy, Proc. Indiana Acad. Sci., 1994, 106, 531-538.

132 A. J. H. M. Meijer and E. M. Goldfield, Phys. Chem. Chem. Phys., 2001, 3, 2811-2818.

133 R. Padmanaban and S. Mahapatra, J. Phys. Chem. A, 2006, 110, 6039-6046.

134 T.-S. Chu and K.-L. Han, Phys. Chem. Chem. Phys., 2008, 10, 2431-2441.

135 A. K. Tiwari, S. Kolakkandy and N. Sathyamurthy, J. Phys. Chem. A, 2009, 113, 9568-9574. 
136 M. T. Cvitas and S. C. Althorpe, Comput. Phys. Commun., 2007, 177, 357-361.

137 M. Hankel, S. C. Smith, S. K. Gray and G. G. Balint-Kurti, Comput. Phys. Commun., 2008, 179, 569-578.

138 S. Adhikari and A. J. C. Varandas, Comput. Phys. Commun., 2013, 184, 270-283.

139 A. J. H. M. Meijer and E. M. Goldfield, J. Chem. Phys., 2000, 113, 11055-11062.

140 J. von Neumann and E. Wigner, Phys. Z., 1929, 30, 465-467.

141 R. Renner, Z. Phys., 1934, 92, 172-193.

142 E. Teller, J. Phys. Chem., 1937, 41, 109-116.

143 H. A. Jahn and E. Teller, Proc. R. Soc. London, Ser. A, 1937, 161, 220-235.

144 H. C. Longuet-Higgins, U. Opik, M. H. L. Pryce and R. A. Sack, Proc. R. Soc. London, Ser. A, 1958, 244, 1-16.

145 G. Herzberg and H. C. Longuet-Higgins, Discuss. Faraday Soc., 1963, 35, 77-82.

146 H. C. Longuet-Higgins, in Advances in Spectroscopy, ed. H. W. Thompson, Interscience, New York, 1961, vol. II; H. C. Longuet-Higgins, Proc. Roy. Soc. London, Ser. A, 1975, 344, 147-156.

147 M. D. Sturge, Solid State Phys., 1967, 20, 91-211.

148 R. Englman, The Jahn-Teller Effect in Molecules and Crystals, Wiley, New York, 1972.

149 T. Carrington, Discuss. Faraday Soc., 1972, 53, 27-34; T. Carrington, Acc. Chem. Res., 1974, 7, 20-25.

150 C. A. Mead and D. G. Truhlar, J. Chem. Phys., 1979, 70, 2284-2296.

151 H. Köppel, W. Domcke and L. S. Cederbaum, Adv. Chem. Phys., 1984, 57, 59-246.

152 I. B. Bersuker, The Jahn-Teller Effect and Vibronic interactions in Modern Chemistry, Plenum Press, New York, 1984; I. B. Bersuker and V. Z. Polinger, Vibronic interactions in Molecules and Crystals, Springer-Verlag, Berlin, 1989.

153 G. Fischer, Vibronic coupling, Academic Press, New York, 1984.

154 R. L. Whetten, G. S. Ezra and E. R. Grant, Annu. Rev. Phys. Chem., 1985, 36, 277-320.

155 B. H. Lengsfield and D. R. Yarkony, Adv. Chem. Phys., 1992, 82, 1-71.

156 W. Domcke and G. Stock, Adv. Chem. Phys., 1997, 100, 1-169; F. Bernadi, M. Olivucci and M. A. Robb, Chem. Soc. Rev., 1996, 25, 321-328.

157 Conical intersections in Photochemistry, Spectroscopy and Chemical Dynamics, in Chemical Physics, ed. Y. Hass, M. Klessinger and S. Zilberg, Elsevier, 2000, vol. 259.

158 G. A. Worth and M. A. Robb, Adv. Chem. Phys., 2002, 124, 355-431.

159 G. A. Worth and L. S. Cederbaum, Annu. Rev. Phys. Chem., 2004, 55, 127-158.

160 Conical Intersections: Theory, Computation and Experiment, ed. W. Domcke, D. R. Yarkony and H. Köppel, World Scientific, Singapore, 2011, vol. 17.

161 M. Born and R. Oppenheimer, Ann. Phys., 1927, 84, 457-484. 162 M. Born and K. Huang, Dynamical theory of Crystal Lattices, Oxford University Press, New York, 1954.
163 W. Pauli Jr, Ann. Phys., 1922, 68, 177-240; P. Güttinger, Z. Phys., 1932, 73, 169-184; H. G. A. Hellmann, Z. Phys., 1933, 85, 180-190; R. P. Feynman, Phys. Rev., 1939, 56, 340-343.

164 W. Lichten, Phys. Rev., 1963, 131, 229-238.

165 F. T. Smith, Phys. Rev., 1969, 179, 111-123.

166 M. Baer, Chem. Phys. Lett., 1975, 35, 112-118; M. Baer, Mol. Phys., 1980, 40, 1011-1013.

167 Z. H. Top and M. Baer, J. Chem. Phys., 1977, 66, 1363-1371.

168 E. F. van Dishoeck, M. C. van Hemert, A. C. Allison and A. Dalgarno, J. Chem. Phys., 1984, 81, 5709-5724.

169 C. A. Mead and D. G. Truhlar, J. Chem. Phys., 1982, 77, 6090-6098.

170 T. F. O’Malley, Adv. At. Mol. Phys., 1971, 7, 223-249; C. I. Ballhausen and A. E. Hansen, Annu. Rev. Phys. Chem., 1972, 23, 15-38; V. Sidis, Adv. Chem. Phys., 1992, 82, 73-134; M. Baer, Adv. Chem. Phys., 1992, 82, 187-241.

171 T. Pacher, L. S. Cederbaum and H. Köppel, Adv. Chem. Phys., 1993, 84, 293-391.

172 H. Köppel, in ref. 77, pp. 175-204.

173 A. Thiel and H. Köppel, J. Chem. Phys., 1999, 110, 9371-9383.

174 H. Köppel, J. Gronki and S. Mahapatra, J. Chem. Phys., 2001, 115, 2377-2388.

175 D. R. Yarkony, Acc. Chem. Res., 1998, 31, 511-518.

176 S. Mahapatra, Acc. Chem. Res., 2009, 42, 1004-1015.

177 C. A. Mead, Rev. Mod. Phys., 1992, 64, 51-85.

178 M. V. Berry, Proc. R. Soc. London, Ser. A, 1984, 392, 45-57.

179 S. Pancharatnam, Proc. - Indian Acad. Sci., Sect. A, 1956, 44, 247-262.

180 B. K. Kendrick, in ref. 77, pp. 521-553.

181 M. Baer, J. Chem. Phys., 1997, 107, 2694-2695.

182 M. Baer, J. Chem. Phys., 1997, 107, 10662-10666.

183 U. Manthe and H. Köppel, J. Chem. Phys., 1990, 93, 345-356; U. Manthe and H. Köppel, J. Chem. Phys., 1990, 93, 1658-1669; U. Manthe, H. Köppel and L. S. Cederbaum, J. Chem. Phys., 1991, 95, 1708-1720.

184 S. Mahapatra, H. Köppel and L. S. Cederbaum, J. Phys. Chem. A, 2001, 105, 2321-2329.

185 S. Mahapatra, in ref. 77, pp. 555-581.

186 B. Maiti, S. Mahapatra and N. Sathyamurthy, J. Chem. Phys., 2000, 113, 59-66.

187 B. Maiti and N. Sathyamurthy, Chem. Phys. Lett., 2001, 345, 461-470.

188 P. Bhatia, B. Maiti, N. Sathyamurthy, S. Stamatiadis and S. C. Farantos, Phys. Chem. Chem. Phys., 1999, 1, 1105-1113; B. Maiti, N. Sathyamurthy, S. Stamatiadis and S. C. Farantos, Indian J. Chem., Sect. A: Inorg., Bio-inorg., Phys., Theor. Anal. Chem., 2000, 39, 338-344.

189 D. Koner, J. C. S. V. Veliz, A. van der Avoird and M. Meuwly, Phys. Chem. Chem. Phys., 2019, 21, 24976-24983.

190 A. N. Panda and N. Sathyamurthy, J. Phys. Chem. A, 2003, 107, 7125-7131.

191 J. S. Lee and D. Secrest, J. Chem. Phys., 1986, 85, 6565-6575.

192 D. Koner, M. Schwilk, S. Patra, E. J. Bieske and M. Meuwly, 2020, arXiv:2004.12404v1. 
193 J. Tennyson, M. A. Kostin, P. Barletta, G. J. Harris, O. L. Polyansky, J. Ramanlal and N. F. Zobov, Comput. Phys. Commun., 2004, 163, 85-116.

194 F. J. Aoiz, L. Bañares and V. J. Herrero, Int. Rev. Phys. Chem., 2005, 24, 119-190.

195 J. Stärck and W. Meyer, Chem. Phys., 1993, 176, 83-95.

196 S. Mahapatra, N. Sathyamurthy, S. Kumar and F. A. Gianturco, Chem. Phys. Lett., 1995, 241, 223-228.

197 S. Mahapatra and N. Sathyamurthy, J. Phys. Chem., 1996, 100, 2759-2761.

198 A. N. Panda and N. Sathyamurthy, J. Chem. Phys., 2004, 121, 9343-9351.

199 D. Skouteris, J. F. Castillo and D. E. Manolopoulos, Comput. Phys. Commun., 2000, 133, 128-135.

200 R. Jaquet and M. Heinen, J. Phys. Chem., 2001, 105, 2738-2747.

201 S. Mahapatra, Phys. Chem. Chem. Phys., 2000, 2, 671-677.

202 A. N. Panda, K. Giri and N. Sathyamurthy, J. Phys. Chem. A, 2005, 109, 2057-2061.

203 M. Zimmer and F. Linder, Chem. Phys. Lett., 1992, 195, 153-158; M. Zimmer and F. Liner, J. Phys. B: At., Mol. Opt. Phys., 1995, 28, 2671-2687.

204 M. S. Huq, L. D. Doverspike and R. L. Champion, Phys. Rev. A: At., Mol., Opt. Phys., 1982, 27, 2831-2839.

205 E. Haufler, S. Schlemmer and D. Gerlich, J. Phys. Chem. A, 1997, 101, 6441-6447.

206 K. Giri and N. Sathyamurthy, J. Phys. Chem. A, 2006, 110, 13843-13849.

207 C. Stroud, N. Sathyamurthy, R. Rangarajan and L. M. Raff, Chem. Phys. Lett., 1977, 48, 350-353.

208 N. Balakrishnan and N. Sathyamurthy, Comput. Phys. Commun., 1991, 63, 209-215.

209 D. R. McLaughlin and D. L. Thompson, J. Chem. Phys., 1979, 70, 2748-2769.

210 T. Joseph and N. Sathyamurthy, J. Chem. Phys., 1987, 86, 704-714.

211 N. Balakrishnan and N. Sathyamurthy, Chem. Phys. Lett., 1993, 201, 294-300.

212 N. Balakrishnan and N. Sathyamurthy, Chem. Phys. Lett., 1995, 240, 119-124.

213 C. Lanczos, J. Res. Natl. Bur. Stand., 1950, 45, 255-282.

214 T. J. Park and J. C. Light, J. Chem. Phys., 1986, 85, 5870-5876.

215 K. Sakimoto and K. Onda, Chem. Phys. Lett., 1994, 226, 227-234.

216 N. Sathyamurthy, T. Joseph and M. Baer, Chem. Phys., 1987, 114, 73-83.

217 V. Balasubramanian, B. K. Mishra, A. Bahel, S. Kumar and N. Sathyamurthy, J. Chem. Phys., 1991, 95, 4160-4167.

218 S. Mahapatra and N. Sathyamurthy, J. Chem. Phys., 1995, 102, 6057-6066.

219 S. Mahapatra, N. Sathyamurthy and R. Ramaswamy, Pramana, 1997, 48, 411-424.

220 S. Mahapatra, R. Ramaswamy and N. Sathyamurthy, J. Chem. Phys., 1996, 104, 3989-3997.

221 S. Mahapatra and N. Sathyamurthy, J. Chem. Phys., 1996, 105, 10934-10943.
222 S. Mahapatra and N. Sathyamurthy, J. Chem. Phys., 1997, 107, 6621-6626.

223 C. Kalyanaraman, D. C. Clary and N. Sathyamurthy, J. Chem. Phys., 1999, 111, 10910-10918.

224 B. Maiti, C. Kalyanaraman, A. N. Panda and N. Sathyamurthy, J. Chem. Phys., 2002, 117, 9719-9726.

225 W. A. Chupka, M. E. Russell and K. Refaey, J. Chem. Phys., 1968, 48, 1518-1527.

226 W. A. Chupka and M. E. Russell, J. Chem. Phys., 1968, 49, 5426-5437.

227 A. N. Panda and N. Sathyamurthy, J. Chem. Phys., 2005, 122, 054304.

228 P. Palmieri, C. Puzzarini, V. Aquilanti, G. Capecchi, S. Cavalli, D. De Fazio, A. Aguilar, X. Giménez and J. M. Lucas, Mol. Phys., 2000, 98, 1835-1849.

229 A. K. Tiwari, A. N. Panda and N. Sathyamurthy, J. Phys. Chem. A, 2006, 110, 389-395.

230 A. K. Tiwari and N. Sathyamurthy, J. Phys. Chem. A, 2006, 110, 11200-11207.

231 C. N. Ramachandran, D. De Fazio, S. Cavalli, F. Tarantelli and V. Aquilanti, Chem. Phys. Lett., 2009, 469, 26-30.

232 W. A. Chupka, J. Berkowitz and M. E. Russell, Sixth International Conference on the Physics of Electronic and Atomic Collisions, MIT, Cambridge, 1969, p. 71.

233 W. A. Chupka, in Ion-molecule reactions, ed. J. L. Franklin, Plenum, New York, 1972, ch. 3, vol. 1, p. 72.

234 D. Yuan, Y. Guan, W. Chen, H. Zhao, S. Yu, C. Luo, Y. Tan, T. Xie, X. Wang, Z. Sun and D. H. Zhang, Science, 2018, 362, 1289-1293; S. Zhang, Z. Tan, H. Zhang, Y. Zhang and J. Z. H. Zhang, Chem. Phys., 2000, 255, 397-402; D. Yuan, S. Yu, W. Chen, J. Sang, C. Luo, T. Wang, X. Xu, P. Casavecchia, X. Wang, Z. Sun, D. H. Zhang and X. Yang, Nat. Chem., 2018, 10, 653-658.

235 D. Yuan, W. Chen, C. Luo, Y. Tan, S. Li, Y. Huang, Z. Sun, $\mathrm{X}$. Yang and X. Wang, J. Phys. Chem. Lett., 2020, 11, 1222-1227; N. C.-M. Bartlett, J. Jankunas, T. Goswami, R. N. Zare, F. Bouakline and S. C. Althorpe, Phys. Chem. Chem. Phys., 2011, 13, 8175-8179; H. Gao, M. Sneha, F. Bouakline, S. C. Althorpe and R. N. Zare, J. Phys. Chem. A, 2015, 119, 12036-12042; J. Jankunas, M. Sneha, R. N. Zare, F. Bouakline and S. C. Althorpe, J. Chem. Phys., 2013, 138, 094310; S. Sukiasyan and H.-D. Meyer, J. Chem. Phys., 2002, 116, 10641-10647.

236 D. Neuhauser, R. S. Judson, R. L. Jaffe, M. Baer and D. J. Kouri, Chem. Phys. Lett., 1991, 176, 546-550; D. H. Zhang, S.-Y. Lee and M. Baer, J. Chem. Phys., 2000, 112, 9802-9809.

237 T. Peng, D. H. Zhang, J. Z. H. Zhang and R. Schinke, Chem. Phys. Lett., 1996, 248, 37-42; J. Dai, J. Chem. Phys., 1997, 107, 4934-4942; G. G. Balint-Kurti, A. I. Gonzalez, E. M. Goldfield and S. K. Gray, Faraday Discuss., 1998, 110, 169-183; S. K. Gray, E. M. Goldfield, G. C. Schatz and G. G. Balint-Kurti, Phys. Chem. Chem. Phys., 1999, 1, 1141-1148; M. Hankel, G. G. Balint-Kurti and S. K. Gray, J. Chem. Phys., 2000, 113, 9658-9677; T. Carroll and E. Goldfield, J. Phys. Chem. A, 2001, 105, 2251-2256; 
S. Y. Lin and H. Guo, Chem. Phys. Lett., 2004, 385, 193-197; S. Y. Lin and H. Guo, J. Chem. Phys., 2008, 129, 124311.

238 T. González-Lezana, P. Larrégaray, L. Bonnet, Y. Wu and W. Bian, J. Chem. Phys., 2018, 148, 234305; Z. Shen, J. Cao and W. Bian, J. Chem. Phys., 2015, 142, 164309; Z.-P. Sun, W.-K. Zhao and C.-L. Yang, J. Chem. Phys., 2017, 117, e25431; L. Mouret, J.-M. Launay, M. Terao-Dunseath and K. Dunseath, Phys. Chem. Chem. Phys., 2004, 6, 4105-4110; S. Y. Lin and H. Guo, J. Chem. Phys., 2003, 119, 11602-11608; S. Y. Lin and H. Guo, J. Phys. Chem. A, 2004, 108, 2141-2148; S. Y. Lin and H. Guo, J. Chem. Phys., 2004, 121, 1285-1292.

239 S. Ghosh, R. Sharma, S. Adhikari and A. J. C. Varandas, Phys. Chem. Chem. Phys., 2019, 21, 20166-20176; S. Y. Lin, H. Guo, P. Honvault and D. Xie, J. Phys. Chem. A, 2006, 110, 23641-23643; P. Bargueño, T. González-Lezana, P. Larrégaray, L. Bonnet and J. C. Rayez, Phys. Chem. Chem. Phys., 2007, 9, 1127-1137; S. Y. Lin, H. Guo, G. Lendvay and D. Xie, Phys. Chem. Chem. Phys., 2009, 11, 4715-4721; S. Y. Lin, E. J. Rackham and H. Guo, J. Phys. Chem. A, 2006, 110, 1534-1540; S. Y. Lin, Z. Sun, H. Guo, D. H. Zhang, P. Honvault, D. Xie and S.-Y. Lee, J. Phys. Chem. A, 2008, 112, 602-611; Z. Sun, D. H. Zhang, C. Xu, S. Zhou, D. Xie, G. Lendvay, S.-Y. Lee, S. Y. Lin and H. Guo, J. Am. Chem. Soc., 2008, 130, 14962-14963; M. Hankel, S. C. Smith and A. J. H. M. Meijer, J. Chem. Phys., 2007, 127, 064316.

240 P. Defazio, S. K. Gray, C. Petrongolo and C. Oliva, J. Chem. Phys., 2001, 115, 3208-3214; P. Defazio, C. Petrongolo, C. Oliva, M. González and R. Sayós, J. Chem. Phys., 2002, 117, 3647-3655.

241 P. Bargueño, P. G. Jambrina, J. M. Alvariño, M. Menéndez, E. Verdasco, M. Hankel, S. C. Smith, F. J. Aoiz and T. González-Lezana, Phys. Chem. Chem. Phys., 2011, 13, 8502-8514; H. Yang, K.-L. Han, S. Nanbu, G. G. BalintKurti, H. Zhang, S. C. Smith and M. Hankel, J. Theor. Comput. Chem., 2009, 8, 1003-1024; H. Yang, K.-L. Han, S. Nanbu, H. Nakamura, G. G. Balint-Kurti, H. Zhang, S. C. Smith and M. Hankel, J. Chem. Phys., 2008, 128, 014308; M. Bittererová, J. M. Bowman and K. Peterson, J. Chem. Phys., 2000, 113, 6186-6196; M. Bittererová and J. M. Bowman, J. Chem. Phys., 2000, 113, 1-3; S. Y. Lin, K.-L. Han and J. Z. H. Zhang, Chem. Phys. Lett., 2000, 324, 122-126.

242 B.-Y. Tang, B.-H. Yang, K.-L. Han, R.-Q. Zhang and J. Z. H. Zhang, J. Chem. Phys., 2000, 113, 10105-10113; P. Defazio and C. Petrongolo, J. Phys. Chem. A, 2009, 113, 4208-4212; A. Li, H. Guo, Z. Sun, J. Kłos and M. H. Alexander, Phys. Chem. Chem. Phys., 2013, 15, 15347-15355; N. Bulut, J. Kłos and M. H. Alexander, J. Chem. Phys., 2012, 136, 104304.

243 M.-D. Chen, B.-Y. Tang, K.-L. Han, N.-Q. Lou and J. Z. H. Zhang, J. Chem. Phys., 2003, 118, 6852-6857; C. Xie, A. Li, D. Xie and H. Guo, J. Chem. Phys., 2011, 135, 164312; N. Bulut, O. Roncero, M. Jorfi and P. Honvault, J. Chem. Phys., 2011, 135, 104307; A. Li,
C. Xie, D. Xie and H. Guo, J. Chem. Phys., 2011, 134, 194309; M.-H. Ge, T.-S. Chu and K.-L. Han, J. Theor. Comput. Chem., 2008, 7, 607-613.

244 S. Y. Lin, H. Guo and P. Honvault, Chem. Phys. Lett., 2008, 453, 140-144; N. Bulut, A. Zanchet, P. Honvault, B. BusseryHonvault and L. Bañares, J. Chem. Phys., 2009, 130, 194303; M. Jorfi, P. Honvault, B. Bussery-Honvault, L. Bañares and N. Bulut, Mol. Phys., 2011, 109, 543-550; A. Zanchet, T. González-Lezana, O. Roncero, M. Jorfi, P. Honvault and M. Hankel, J. Chem. Phys., 2012, 136, 164309; T. Rajagopala Rao, S. Goswami, S. Mahapatra, B. Bussery-Honvault and P. Honvault, J. Chem. Phys., 2012, 136, 094318; S. Goswami, B. Bussery-Honvault, P. Honvault and S. Mahapatra, Mol. Phys., 2017, 115, 2658-2672; S. Goswami, J. Sahoo, T. Rajagopala Rao, B. Bussery-Honvault, P. Honvault and S. Mahapatra, Eur. Phys. J. D, 2018, 72, 225.

245 S. Goswami, T. Rajagopala Rao, S. Mahapatra, B. BusseryHonvault and P. Honvault, J. Phys. Chem. A, 2014, 118, 5915-5926.

246 D. Wang, J. R. Stallcop, W. M. Huo, C. E. Dateo, D. W. Schwenke and H. Partridge, J. Chem. Phys., 2003, 118, 2186-2189; D. Wang, W. M. Huo, C. E. Dateo, D. W. Schwenke and J. R. Stallcop, J. Chem. Phys., 2004, 120, 6041-6050; Y. Wang, F. Meng, P. Yan and D. Wang, Chem. Phys. Lett., 2015, 633, 202-207; E. Garcia, A. Laganà and D. Skouteris, Phys. Chem. Chem. Phys., 2012, 14, 1589-1595; D. Skouteris, L. Pacifici and A. Laganà, Mol. Phys., 2004, 102, 2237-2248.

247 K.-L. Yeh, D. Xie, D. H. Zhang, S.-Y. Lee and R. Schinke, J. Phys. Chem. A, 2003, 107, 7215-7219; C. H. Yuen, D. Lapierre, F. Gatti, V. Kokoouline and V. G. Tyuterev, J. Phys. Chem. A, 2019, 123, 7733-7743; Y. Li, Z. Sun, B. Jiang, D. Xie, R. Dawes and H. Guo, J. Chem. Phys., 2014, 141, 081102; W. Xie, L. Liu, Z. Sun, H. Guo and R. Dawes, J. Chem. Phys., 2015, 142, 064308; Z. Sun, D. Yu, W. Xie, J. Hou, R. Dawes and H. Guo, J. Chem. Phys., 2015, 142, 174312; Z. Sun, L. Liu, S. Y. Lin, R. Schinke, H. Guo and D. H. Zhang, Proc. Natl. Acad. Sci. U. S. A., 2010, 107, 555-558.

248 F. Gogtas, G. G. Balint-Kurti and A. Offer, J. Chem. Phys., 1996, 104, 7927-7939; A. Aguado, M. Paniagua, M. Lara and O. Roncero, J. Chem. Phys., 1997, 106, 1013-1025; W. Zhu, D. Wang and J. Z. H. Zhang, Theor. Chem. Acc., 1997, 96, 31-38; M. Lara, A. Aguado, M. Paniagua and O. Roncero, J. Chem. Phys., 2000, 113, 1781-1794; W.-T. Lia, M.-D. Chen and Z. Sun, Chin. J. Chem. Phys., 2015, 28, 415-425; X. Liu, C. Xie and H. Guo, Chem. Phys., 2018, 509, 66-71.

249 J. Song and Z. Zhu, Comput. Theor. Chem., 2020, 1173, 112703; D. He, J. Yuan, H. Li and M. Chen, Sci. Rep., 2016, 6, 25083; D. He, J. Yuan and M. Chen, Sci. Rep., 2017, 7, 3084.

250 R. Padmanaban and S. Mahapatra, J. Chem. Phys., 2002, 117, 6469-6477; R. Padmanaban and S. Mahapatra, J. Chem. Phys., 2004, 121, 7681-7691; R. Padmanaban and S. Mahapatra, J. Phys. Chem. A, 2006, 110, 
6039-6046; T. Roy and S. Mahapatra, J. Chem. Phys., 2012, 136, 174313.

251 T. Roy, T. Rajagopala Rao and S. Mahapatra, Chem. Phys. Lett., 2011, 501, 252-256.

252 B. Jayachander Rao and S. Mahapatra, J. Chem. Phys., 2007, 127, 244307.

253 X. He, W. Li, H. Meng, C. Li, G. Guo, X. Qiu and J. Wei, Phys. Chem. Chem. Phys., 2019, 21, 7196-7207, and references therein.

254 S. Bhattacharya and A. N. Panda, J. Phys. B: At., Mol. Opt. Phys., 2009, 42, 085201.

255 D. Koner and A. N. Panda, J. Phys. Chem. A, 2013, 117, 13070-13078; D. Koner, L. Barrios, T. González-Lezana and A. N. Panda, J. Chem. Phys., 2014, 141, 114302.

256 D. Koner, L. Barrios, T. González-Lezana and A. N. Panda, J. Phys. Chem. A, 2015, 119, 12052-12061.

257 D. Koner, L. Barrios, T. González-Lezana and A. N. Panda, J. Chem. Phys., 2016, 144, 034303.

258 A. N. Panda and S. C. Althorpe, Chem. Phys. Lett., 2007, 439, 50-54.

259 A. N. Panda, J. Phys. Chem. A, 2008, 112, 5327-5331.

260 B. Zhao, Z. Sun and H. Guo, J. Chem. Phys., 2016, 145, 184106; C. Xiao, X. Xu, S. Liu, T. Wang, W. Dong, T. Yang, Z. Sun, D. Dai, X. Xu, D. H. Zhang and X. Yang, Science, 2011, 333, 440-442; S. Liu, C. Xiao, T. Wang, J. Chen, T. Yang, X. Xu, D. H. Zhang and X. Yang, Faraday Discuss., 2012, 157, 101-111; S. Liu, X. Xu and D. H. Zhang, J. Chem. Phys., 2012, 136, 144302; B. Zhao, Z. Sun and H. Guo, J. Chem. Phys., 2016, 145, 134308; Z. Zhao, S. Liu and D. H. Zhang, J. Chem. Phys., 2016, 145, 134301.

261 S. Liu and D. H. Zhang, Chem. Sci., 2016, 7, 261-265; D. H. Zhang and J. C. Light, J. Chem. Soc., Faraday Trans., 1997, 93, 691-697; D. H. Zhang, M. A. Collins and S.-Y. Lee, Science, 2000, 290, 961-963; B. Fu and D. H. Zhang, J. Chem. Phys., 2013, 138, 184308; B. Fu and D. H. Zhang, J. Chem. Phys., 2015, 142, 064314; Z.-q. Zhao, S. Liu and D. H. Zhang, Chin. J. Chem. Phys., 2017, 30, 16-24.

262 D. M. Medvedev, S. K. Gray, E. M. Goldfield, M. J. Lakin, D. Troya and G. C. Schatz, J. Chem. Phys., 2004, 120, 1231-1238; R. Valero, D. A. McCormack and G.-J. Kroes, J. Chem. Phys., 2004, 120, 4263-4272; R. Valero and G.-J. Kroes, J. Phys. Chem. A, 2004, 108, 8672-8681; S. Liu, X. Xu and D. H. Zhang, J. Chem. Phys., 2011, 135, 141108; S. Liu, X. Xu and D. H. Zhang, Theor. Chem. Acc., 2012, 131, 1068; J. Ma, J. Li and H. Guo, J. Phys. Chem. Lett., 2012, 3, 2482-2486; C. Wang, S. Liu and D. H. Zhang, Chem. Phys. Lett., 2012, 537, 16-20; S. Liu, J. Chen, B. Fu and D. H. Zhang, Theor. Chem. Acc., 2014, 133, 1558; D. H. Zhang, J. Chem. Phys., 2006, 125, 133102.

263 W. Zhu, J. Z. H. Zhang, Y. C. Zhang, Y. B. Zhang, L. X. Zhan, S. L. Zhang and D. H. Zhang, J. Chem. Phys., 1998, 108, 3509-3516; S. Bhattacharya, A. Kirwai, A. N. Panda and H.-D. Meyer, J. Chem. Sci., 2012, 124, 65-73; J. Z. H. Zhang and S.-Y. Lee, J. Chem. Phys., 2000, 112, 203-211.

264 B. Jiang and H. Guo, J. Chem. Phys., 2013, 139, 224310; W.-Y. Ma, K.-L. Han and J. Z. H. Zhang, J. Chem. Phys.,
2002, 117, 5642-5646; W.-Y. Ma, K.-L. Han, M. L. Wang and J. Z. H. Zhang, J. Chem. Phys., 2002, 117, 172-176.

265 R. Liu, H. Xiong and M. Yang, J. Chem. Phys., 2012, 137, 174113; W. Zhang, Y. Zhou, G. Wu, Y. Lu, H. Pan, B. Fu, Q. Shuai, L. Liu, S. Liu, L. Zhang, B. Jiang, D. Dai, S.-Y. Lee, Z. Xie, B. J. Braams, J. M. Bowman, M. A. Collins, D. H. Zhang and X. Yang, Proc. Natl. Acad. Sci. U. S. A., 2010, 107, 12782-12785; Z. Zhao, Z. Zhang, S. Liu and D. H. Zhang, Nat. Commun., 2017, 8, 14506; M. Yang, D. H. Zhang and S.-Y. Lee, J. Chem. Phys., 2002, 117, 9539-9542; Y. Zhou, C. Wang and D. H. Zhang, J. Chem. Phys., 2011, 135, 024313; S. Liu, J. Chen, Z. Zhang and D. H. Zhang, J. Chem. Phys., 2013, 138, 011101; Z. Zhang, J. Chen, S. Liu and D. H. Zhang, J. Chem. Phys., 2014, 140, 224304; Z. Zhang and D. H. Zhang, J. Chem. Phys., 2014, 141, 144309.

266 M. Yang, S.-Y. Lee and D. H. Zhang, J. Chem. Phys., 2007, 126, 064303; R. Liu, M. Yang, G. Czakó, J. M. Bowman, J. Li and H. Guo, J. Phys. Chem. Lett., 2012, 3, 3776-3780; M.-L. Wang, Y.-M. Li and J. Z. H. Zhang, J. Phys. Chem. A, 2001, 105, 2530-2534; W. Yan, F. Meng and D. Wang, J. Phys. Chem. A, 2013, 117, 12236-12242.

267 Z. Zhang, Y. Zhou, D. H. Zhang, G. Czakó and J. M. Bowman, J. Phys. Chem. Lett., 2012, 3, 3416-3419; R. Liu, F. Wang, B. Jiang, G. Czakó, M. Yang, K. Liu and H. Guo, J. Chem. Phys., 2014, 141, 074310; N. Liu and M. Yang, J. Chem. Phys., 2015, 143, 134305.

268 Z. Chen, J. Chen, R. Chen, T. Xie, X. Wang, S. Liu, G. Wu, D. Dai, X. Yang and D. H. Zhang, Proc. Natl. Acad. Sci. U. S. A., 2020, 117, 9202-9207.

269 T.-S. Chu, K.-L. Han and J. Espinosa-Garcia, J. Chem. Phys., 2009, 131, 244303; D. Wang and G. Czakó, J. Phys. Chem. A, 2013, 117, 7124-7130; J. Qi, H. Song, M. Yang, J. Palma, U. Manthe and H. Guo, J. Chem. Phys., 2016, 144, 171101.

270 R. J. Butler, Annu. Rev. Phys. Chem., 1998, 49, 125-171.

271 A. D. Hammerich, U. Manthe, R. Kosloff, H.-D. Meyer and L. S. Cederbaum, J. Chem. Phys., 1994, 101, 5623-5646.

272 C. Woywod, M. Stengle, W. Domcke, H. Flöthmann and R. Schinke, J. Chem. Phys., 1997, 107, 7282-7295; H. Flöthmann, C. Beck, R. Schinke, C. Woywod and W. Domcke, J. Chem. Phys., 1997, 107, 7296-7313; D. Simah, B. Hartke and H.-J. Werner, J. Chem. Phys., 1999, 111, 4523-4534; D. Skouteris, B. Hartke and H.-J. Werner, J. Phys. Chem. A, 2001, 105, 2458-2467.

273 K. Drukker and G. C. Schatz, J. Chem. Phys., 1999, 111, 2451-2463; Y.-T. Hsu, K. Liu, L. A. Pederson and G. C. Schatz, J. Chem. Phys., 1999, 111, 7931-7944; M. R. Hoffmann and G. C. Schatz, J. Chem. Phys., 2000, 113, 9456-9465; X. Liu, J. J. Lin, S. Harich, G. C. Schatz and X. Yang, Science, 2000, 289, 1536-1538; F. J. Aoiz, L. Bañares, J. F. Castillo, V. J. Herrero, B. Martinez-Haya, P. Honvault, J. M. Launay, X. Liu, J. J. Lin, S. Harich, C. C. Wang and X. Yang, J. Chem. Phys., 2002, 116, 10692-10703.

274 S. K. Gray, G. G. Balint-Kurti, G. C. Schatz, J. J. Lin, X. Liu, S. Harich and X. Yang, J. Chem. Phys., 2000, 113, 7330-7344. 
275 T. W. Whiteley, A. J. Dobbyn, J. N. L. Connor and G. C. Schatz, Phys. Chem. Chem. Phys., 2000, 2, 549-556.

276 S.-H. Lee and K. Liu, J. Phys. Chem. A, 1998, 102, 8637-8640; A. S. Zyubin, A. M. Mebel, S. D. Chao and R. T. Skodje, J. Chem. Phys., 2001, 114, 320-330.

277 Y. Zhang, T.-X. Xie, K.-L. Han and J. Z. H. Zhang, J. Chem. Phys., 2003, 119, 12921-12925; Y. Zhang, T.-X. Xie and K.-L. Han, J. Phys. Chem. A, 2003, 107, 10893-10896.

278 M. H. Alexander, G. Capecchi and H.-J. Werner, Faraday Discuss., 2004, 127, 59-72; D. Skouteris, Z. Laganà, G. Capecchi and H.-J. Werner, Phys. Chem. Chem. Phys., 2004, 6, 5000-5006.

279 S. Ghosal and S. Mahapatra, J. Chem. Phys., 2004, 121, 5740-5753; S. Ghosal and S. Mahapatra, J. Photochem. Photobiol., A, 2007, 190, 161-168.

280 X. Wang, W. Dong, C. Xiao, L. Che, Z. Ren, D. Dai, X. Wang, P. Casavecchia, X. Yang, B. Jiang, D. Xie, Z. Sun, S.-Y. Lee, D. H. Zhang, H.-J. Werner and M. H. Alexander, Science, 2008, 322, 573-576.

281 H. Yang, M. Hankel, Y. Zheng and A. J. C. Varandas, J. Chem. Phys., 2011, 135, 024306.

282 T.-S. Chu, T.-X. Xie and K.-L. Han, J. Chem. Phys., 2004, 121, 9352-9360; P. Defazio, P. Gamallo and C. Pentrogolo, J. Chem. Phys., 2012, 136, 054308.

283 S. Wang, Z. Yiang, J. Yuan and M. Chen, Sci. Rep., 2018, 8, 17960 .

284 L. A. Pederson, G. C. Schatz, T. Hollebeek, T.-S. Ho, H. Rabitz and L. B. Harding, J. Phys. Chem. A, 2000, 104, 2301-2307.

285 N. Marković and G. D. Billing, Chem. Phys., 1995, 191, 247-260; T.-S. Chu, A. J. C. Varandas and K.-L. Han, Chem. Phys. Lett., 2009, 471, 222-228; S. Ghosh, T. Sahoo, S. Adhikari, R. Sharma and A. J. C. Varandas, J. Phys. Chem. A, 2015, 119, 12392-12403.

286 T. S. Chu and K.-L. Han, J. Phys. Chem. A, 2005, 109, 2050-2056; R.-F. Lu, T. S. Chu and K.-L. Han, J. Phys. Chem. A, 2005, 109, 6683-6688.

287 B. Zhao and U. Manthe, J. Chem. Phys., 2020, 152, 231102.

288 R. N. Porter, R. M. Stevens and M. Karplus, J. Chem. Phys., 1968, 49, 5163-5178.

289 P. Siegbahn and B. Liu, J. Chem. Phys., 1978, 68, 2457-2465; D. G. Truhlar and C. J. Horowitz, J. Chem. Phys., 1978, 68, 2466-2476; D. G. Truhlar and C. J. Horowitz, J. Chem. Phys., 1979, 71, 1514(E).

290 A. J. C. Varandas, F. B. Brown, C. A. Mead, D. G. Truhlar and N. C. Blais, J. Chem. Phys., 1987, 86, 6258-6269.

291 A. I. Boothroyd, W. J. Keogh, P. G. Martin and M. R. Peterson, J. Chem. Phys., 1991, 95, 4343-4359.

292 A. I. Boothroyd, W. J. Keogh, P. G. Martin and M. R. Peterson, J. Chem. Phys., 1996, 104, 7139-7152.

293 R. Abrol, A. Shaw, A. Kuppermann and D. R. Yarkony, J. Chem. Phys., 2001, 115, 4640-4659.

294 R. Abrol and A. Kuppermann, J. Chem. Phys., 2002, 116, 1035-1062.

295 B. Lepetit, Z. Peng and A. Kuppermann, Chem. Phys. Lett., 1990, 166, 572-580.
296 A. J. C. Varandas and H. G. Yu, Chem. Phys. Lett., 1996, 259, 336-341.

297 R.-F. Lu, T. S. Chu, Y. Zhang, K.-L. Han, A. J. C. Varandas and J. Z. H. Zhang, J. Chem. Phys., 2006, 125, 133108.

298 S. Mahapatra and H. Köppel, J. Chem. Phys., 1998, 109, 1721-1733.

299 R. Bruckmeier, Ch. Wunderlich and H. Figger, Phys. Rev. Lett., 1994, 72, 2550-2553.

300 S. Mahapatra and H. Köppel, Phys. Rev. Lett., 1998, 81, 3116-3119.

301 C. A. Mead, J. Chem. Phys., 1980, 72, 3839-3840.

302 B. Lepetit and A. Kuppermann, Chem. Phys. Lett., 1990, 166, 581-588.

303 Y. M. Wu, A. Kuppermann and B. Lepetit, Chem. Phys. Lett., 1991, 186, 319-328.

304 A. Kuppermann and Y.-S. M. Wu, Chem. Phys. Lett., 1993, 205, 577-586.

305 A. Kuppermann and Y.-S. M. Wu, Chem. Phys. Lett., 1995, 241, 229-240.

306 A. Kuppermann and Y.-S. M. Wu, Chem. Phys. Lett., 2001, 349, 537-546.

307 L. Schnieder, K. Seekamp-Rahn, J. Borkowski, E. Wrede, K. Welge, F. J. Aoiz, L. Bañares, M. J. D’Mello, V. J. Herrero, V. Sáez Rábanos and R. E. Wyatt, Science, 1995, 269, 207-210; E. Wrede, L. Schnieder, K. H. Welge, F. J. Aoiz, L. Bañares, J. F. Castillo, B. Martínez-Haya and V. J. Herrero, J. Chem. Phys., 1999, 110, 9971-9981.

308 S. C. Althorpe, F. Fernández-Alonso, B. D. Bean, J. D. Ayres, A. E. Pomerantz, R. N. Zare and E. Wrede, Nature, 2002, 416, 67-70.

309 S. A. Harich, D. Dai, C. C. Wang, X. Yang, S. D. Chao and R. T. Skodje, Nature, 2002, 419, 281-284.

310 D. Dai, C. C. Wang, S. A. Harich, X. Wang, X. Yang, S. D. Chao and R. T. Skodje, Science, 2003, 300, 1730-1734.

311 K. Koszinowski, N. T. Goldberg, J. Zhang, R. N. Zare, F. Bouakline and S. C. Althorpe, J. Chem. Phys., 2007, 127, 124315.

312 K. Koszinowski, N. T. Goldberg, A. E. Pomerantz, R. N. Zare, J. C. Juanes-Marcos and S. C. Althorpe, J. Chem. Phys., 2005, 123, 054306.

313 B. K. Kendrick, J. Chem. Phys., 2000, 112, 5679-5704; B. K. Kendrick, J. Phys. Chem. A, 2003, 107, 6739-6756; B. K. Kendrick, J. Chem. Phys., 2003, 118, 10502-10522.

314 J. C. Juanes-Marcos and S. C. Althorpe, J. Chem. Phys., 2005, 122, 204324.

315 F. Bouakline, S. C. Althorpe and D. Peláez Ruiz, J. Chem. Phys., 2008, 128, 124322.

316 D. Yuan, Y. Guan, W. Chen, H. Zhao, S. Yu, C. Luo, Y. Tan, T. Xie, X. Wang, Z. Sun, D. H. Zhang and X. Yang, Science, 2018, 362, 1289-1293; Y. Xie, H. Zhao, Y. Wang, Y. Huang, T. Wang, X. Xu, C. Xiao, Z. Sun, D. H. Zhang and X. Yang, Science, 2020, 368, 767-771.

317 B. Jayachander Rao and S. Mahapatra, J. Chem. Sci., 2009, 121, 789-795.

318 R. Padmanaban and S. Mahapatra, J. Theor. Comput. Chem., 2006, 5, 871-885. 
319 B. Jayachander Rao, R. Padmanaban and S. Mahapatra, Chem. Phys., 2007, 333, 135-147.

320 T. Rajagopala Rao, B. Jayachander Rao and S. Mahapatra, Chem. Phys., 2009, 365, 129-137; S. Ghosal, B. Jayachander Rao and S. Mahapatra, J. Chem. Sci., 2007, 119, 401-407.

321 G. W. Johnston, B. Katz, K. Tsukiyama and R. Bersohn, J. Phys. Chem., 1987, 91, 5445-5451; A. A. Westenberg and N. De Haas, J. Chem. Phys., 1967, 47, 1393-1405.

322 J. Jankunas, M. Sneha, R. N. Zare, F. Bouakline, S. C. Althorpe, D. Herráez-Aguilar and F. J. Aoiz, Proc. Natl. Acad. Sci. U. S. A., 2014, 111, 15-20.

323 T.-X. Xie, Y. Zhang, M.-Y. Zhao and K.-L. Han, Phys. Chem. Chem. Phys., 2003, 5, 2034-2038; Y. Zhang, T.-X. Xie, K.-L. Han and J. Z. H. Zhang, J. Chem. Phys., 2004, 120, 6000-6004; Y. Zhang, T.-X. Xie, K.-L. Han and J. Z. H. Zhang, J. Chem. Phys., 2006, 124, 134301.

324 D. Skouteris, A. Laganà, G. Capecchi and H.-J. Werner, Phys. Chem. Chem. Phys., 2004, 6, 5000-5006; Z. Sun, D. H. Zhang and M. H. Alexander, J. Chem. Phys., 2010, 132, 034308.

325 B. Jiang, C. Xie and D. Xie, J. Chem. Phys., 2011, 135, 164311; C. Xie, B. Jiang, D. Xie and Z. Sun, J. Chem. Phys., 2012, 136, 114310.

326 S. K. Gray, C. Pentrogolo, K. Drukker and G. C. Schatz, J. Phys. Chem. A, 1999, 103, 9448-9459; Z. Sun, S. Y. Lin and Y. Zheng, J. Chem. Phys., 2011, 135, 234301; T.-S. Chu, X. Zhang and K.-L. Han, J. Chem. Phys., 2005, 122, 214301; S. Y. Lin and H. Guo, J. Phys. Chem. A, 2009, 113, 4285-4293; J. Zhao, J. Chem. Phys., 2013, 138, 134309; B. Han and Y. Zheng, J. Comput. Chem., 2011, 32, 3520-3525.

327 E. J. Heller, J. Chem. Phys., 1975, 62, 1544-1555; E. J. Heller, J. Chem. Phys., 1981, 75, 2923-2931.

328 M. F. Herman and E. A. Kluk, Chem. Phys., 1984, 91, 27-34; W. H. Miller, J. Phys. Chem. A, 2001, 105, 2942-2955; M. Thoss and H. Wang, Annu. Rev. Phys. Chem., 2004, 55, 299-332; E. Martin-Fierro and E. Pollak, J. Chem. Phys., 2007, 126, 164108.

329 A. D. McLachlan, Mol. Phys., 1964, 8, 39-44.

330 G. D. Billing, Chem. Phys. Lett., 1975, 30, 391-393; G. D. Billing, Chem. Phys., 1978, 33, 227-247; N. Balakrishnan and G. D. Billing, J. Chem. Phys., 1996, 104, 4005-4011; S. Adhikari and G. D. Billing, Chem. Phys. Lett., 1999, 309, 249-256; S. Adhikari and G. D. Billing, J. Chem. Phys., 1999, 111, 48-53; R. B. Gerber, V. Buch and M. A. Ratner, J. Chem. Phys., 1982, 77, 3022-3030; J. C. Tully and R. K. Preston, J. Chem. Phys., 1971, 55, 562-572; G. Stock and M. Thoss, Adv. Chem. Phys., 2005, 131, 243-375.

331 R. P. Feynman, Rev. Mod. Phys., 1948, 20, 367-387; N. Makri, Chem. Phys. Lett., 1992, 193, 435-445; S. Krempl, M. Winterstetter, H. Plöhn and W. Domcke, J. Chem. Phys., 1994, 100, 926-937; S. Krempl, M. Winterstetter and W. Domcke, J. Chem. Phys., 1995, 102, 6499-6510; N. Makri and D. E. Makarov, J. Chem. Phys., 1995, 102, 4600-4610.
332 H.-D. Meyer, U. Manthe and L. S. Cederbaum, Chem. Phys. Lett., 1990, 165, 73-78; U. Manthe, H.-D. Meyer and L. S. Cederbaum, J. Chem. Phys., 1992, 97, 3199-3213; M. H. Beck, A. Jaekle, G. A. Worth and H.-D. Meyer, Phys. Rep., 2000, 324, 1-105.

333 H. Wang, M. Thoss and W. H. Miller, J. Chem. Phys., 2001, 115, 2979-2990.

334 J. Frenkel, Wave Mechanics, Clarendon Press, Oxford, UK, 1934.

335 G. Schiffel and U. Manthe, J. Chem. Phys., 2010, 132, 084103; G. Schiffel and U. Manthe, J. Chem. Phys., 2010, 132, 191101; G. Schiffel and U. Manthe, J. Chem. Phys., 2010, 133, 174124; R. Ellerbrock and U. Manthe, J. Chem. Phys., 2017, 147, 241104.

336 H. Wang and M. Thoss, J. Chem. Phys., 2003, 119, 1289-1299. 337 U. Manthe, J. Chem. Phys., 2008, 128, 164116.

338 R. Welsch and U. Manthe, J. Chem. Phys., 2012, 137, 244106.

339 T. Kato and H. Kono, Chem. Phys. Lett., 2004, 392, 533-540; J. Calliat, J. Zanghellini, M. Kitzler, O. Koch, W. Kreuzer and A. Scrinzi, Phys. Rev. A: At., Mol., Opt. Phys., 2005, 71, 012712; M. Nest, T. Klamroth and P. Saalfrank, J. Chem. Phys., 2005, 122, 124102.

340 O. E. Alon, A. I. Strelstov and L. S. Cederbaum, Phys. Rev. A: At., Mol., Opt. Phys., 2008, 77, 033613; O. E. Alon, A. I. Strelstov and L. S. Cederbaum, J. Chem. Phys., 2007, 127, 154103.

341 S. Mandal, S. Ghosh, S. Sardar and S. Adhikari, Int. Rev. Phys. Chem., 2019, 37, 607-700, and references therein.

342 S.-I. Sawada, R. Heather, B. Jackson and H. Metiu, J. Chem. Phys., 1985, 83, 3009-3027; S.-I. Sawada and H. Metiu, J. Chem. Phys., 1986, 84, 227-238.

343 I. Burghardt, H.-D. Meyer and L. S. Cederbaum, J. Chem. Phys., 1999, 111, 2927-2939.

344 I. Burghardt, M. Nest and G. A. Worth, J. Chem. Phys., 2003, 119, 5364-5378; I. Burghardt, K. Giri and G. A. Worth, J. Chem. Phys., 2008, 129, 174104; G. A. Worth, H.-D. Meyer, H. Köppel, L. S. Cederbaum and I. Burghardt, Int. Rev. Phys. Chem., 2008, 27, 569-606; S. Römer, M. Ruckenbauer and I. Burghardt, J. Chem. Phys., 2013, 138, 064106.

345 T. J. Martínez, M. Ben-Nun and R. D. Levine, J. Phys. Chem., 1996, 100, 7884-7895.

346 M. Ben-Nun and T. J. Martínez, J. Chem. Phys., 2000, 112, 6113-6121; M. Ben-Nun and T. J. Martínez, Adv. Chem. Phys., 2002, 121, 439-512; S. Yang, J. D. Coe, B. Kaduk and T. J. Martínez, J. Chem. Phys., 2009, 130, 134113; M. BenNun and T. J. Martínez, Isr. J. Chem., 2007, 47, 75-88.

347 G. A. Worth and I. Burghardt, Chem. Phys. Lett., 2003, 368, 502-508.

348 D. V. Shalashilin and M. S. Child, J. Chem. Phys., 2000, 113, 10028-10036; D. V. Shalashilin and M. S. Child, J. Chem. Phys., 2001, 114, 9296-9304; D. V. Shalashilin and M. S. Child, J. Chem. Phys., 2001, 115, 5367-5375.

349 D. V. Shalashilin and M. S. Child, J. Chem. Phys., 2004, 121, 3563-3568; D. V. Shalashilin and M. S. Child, J. Chem. Phys., 2008, 128, 054102. 
350 D. V. Shalashilin, J. Chem. Phys., 2010, 132, 244111.

351 B. Jackson, Annu. Rev. Phys. Chem., 1995, 46, 251-274.

352 C. Díaz, E. Pijper, R. A. Olsen, H. F. Busnengo, D. J. Auerbach and G.-J. Kroes, Science, 2009, 326, 832-834.

353 N. Gerrits, K. Shakouri, J. Behler and G.-J. Kroes, J. Phys. Chem. Lett., 2019, 10, 1763-1768.

354 A. K. Tiwari, S. Nave and B. Jackson, Phys. Rev. Lett., 2009, 103, 253201.

355 S. Nave, A. K. Tiwari and B. Jackson, J. Phys. Chem. A, 2014, 118, 9615-9631.

356 W. H. Miller, J. Phys. Chem., 1983, 87, 3811-3819.
357 B. Jiang, X. Ren, D. Xie and H. Guo, Proc. Natl. Acad. Sci. U. S. A., 2012, 109, 10224-10227.

358 P. M. Hundt, B. Jiang, M. E. van Reijzen, H. Guo and R. D. Beck, Science, 2014, 344, 504-507.

359 S. Roy, K. J. Nayanthara, N. Tiwari and A. K. Tiwari, Int. Rev. Phys. Chem., 2020, 39, 267-318.

360 L. Zhang and B. Jiang, Phys. Rev. Lett., 2019, 123, 106001. 361 T. Peng, D. H. Zhang, D. Wang, Y. Li and J. Z. H. Zhang, Comput. Phys. Commun., 2000, 128, 492-495.

362 R. V. Krems, Phys. Chem. Chem. Phys., 2019, 21, 13392-13410. 